MATHEMATICS OF COMPUTATION

Volume 69, Number 232, Pages 1409-1434

S $0025-5718(00) 01181-9$

Article electronically published on March 3, 2000

\title{
GALERKIN EIGENVECTOR APPROXIMATIONS
}

\author{
CHRISTOPHER BEATTIE
}

\begin{abstract}
How close are Galerkin eigenvectors to the best approximation available out of the trial subspace? Under a variety of conditions the Galerkin method gives an approximate eigenvector that approaches asymptotically the projection of the exact eigenvector onto the trial subspace - and this occurs more rapidly than the underlying rate of convergence of the approximate eigenvectors. Both orthogonal-Galerkin and Petrov-Galerkin methods are considered here with a special emphasis on nonselfadjoint problems, thus extending earlier studies by Chatelin, Babuška and Osborn, and Knyazev. Consequences for the numerical treatment of elliptic PDEs discretized either with finite element methods or with spectral methods are discussed. New lower bounds to the sep of a pair of operators are developed as well.
\end{abstract}

\section{INTRODUCTION}

Consider the eigenvalue problem for a linear operator $A$ :

$$
\begin{gathered}
\text { Find } \lambda \in \mathbb{C} \text { and } \hat{v} \neq 0 \text { so that } \\
A \hat{v}=\lambda \hat{v} .
\end{gathered}
$$

We seek a family of approximations $\left\{\lambda_{h}, \hat{v}_{h}\right\}_{h>0}$ to the eigenpair $\{\lambda, \hat{v}\}$ using the Galerkin method.

The Galerkin method approximates the operator $A$ with a finite rank operator, $A_{h}$-the "projection" of $A$, that samples the action of $A$ on a given subspace. The solution to (1.1) is then approximated with a matrix eigenvalue problem associated with $A_{h}$.

This work focuses on one particular bit of Galerkin folklore - "the Galerkin method yields an approximate eigenvector for $A$ that is essentially the projection of the exact eigenvector $\hat{v}$ onto the trial subspace" (see Figure 1). We discover that this statement is correct under some mild conditions if 1) "essentially" is taken to mean "asymptotically," and 2) the projection involved is intrinsic to the Galerkin method and may be either orthogonal or oblique depending on how the discretization is organized and what point of view is taken. Results of this nature have been found for self-adjoint operators by Chatelin [3], Babuška and Osborn [2], and Knyazev [10] in various settings. Although more generality is possible, we restrict ourselves to a Hilbert space setting - specific assumptions are found in Section 2.

Received by the editor January 6, 1998 and, in revised form, July 10, 1998 and October 9, 1998.

1991 Mathematics Subject Classification. Primary 65N25; Secondary 65N30, 65F15.

Key words and phrases. Galerkin, eigenvector asymptotics, finite elements, spectral methods, sep.

This work was supported under the auspices of AFOSR Grant F49620-96-1-0329.

(C)2000 American Mathematical Society 


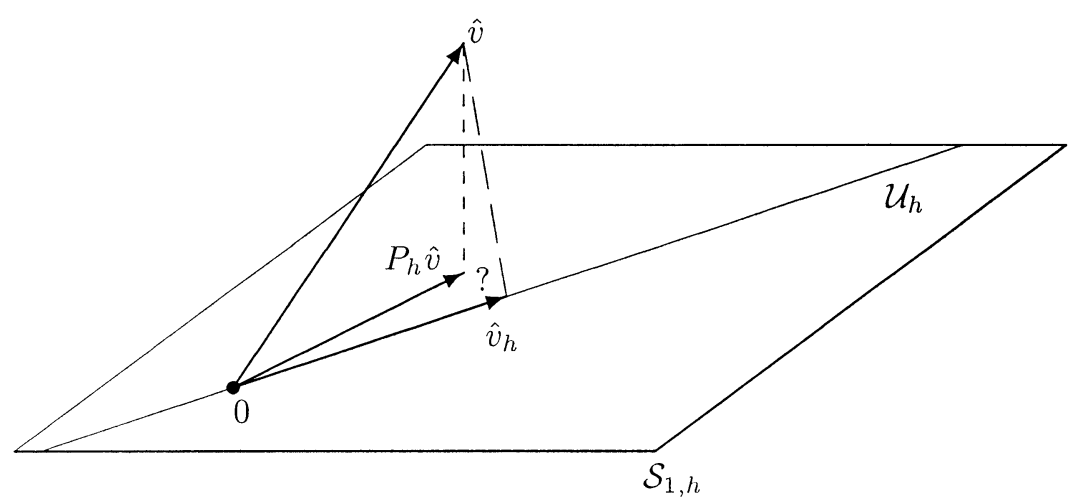

Figure 1 . How close is the approximate eigenvector $\hat{v}_{h}$ to the projected exact eigenvector $P_{h} \hat{v}$ ?

The basic features of Galerkin methods that play a role in our analysis are reviewed in Section 3 Of particular note here is that discussion is not restricted to self-adjoint problems. Section 4 provides analysis for the simplest case-when $A$ is a bounded operator. The case where $A$ is an unbounded operator is considered from two vantage points in the next two sections: with respect to the "energy" norm in Section 5 where a discussion of consequences for the finite element method on elliptic problems may be found; and with respect to the underlying Hilbert space norm in Section 6 where an elliptic problem discretized using a spectral method is discussed. The Appendix contains a development of new lower bounds to the sep of a pair of operators, which plays a role in derivations in the main body of the paper but may also be of independent interest.

\section{Setting of the PRoblem}

2.1. Operators defined via quadratic forms. Although eigenvalue problems are most naturally posed for linear operators, the operators themselves are often difficult to specify fully - particularly with regard to the operator's precise domain of definition. It is often easier to characterize an operator in terms of a quadratic form that is naturally associated with it. This approach usually leads spontaneously to the appropriate choice of underlying Hilbert spaces. The reader may refer to the excellent tract of Kato 9] for background material on quadratic forms.

Let $\mathcal{H}$ be a complex separable Hilbert space with inner product 1 and norm denoted by $\langle\cdot, \cdot\rangle_{\mathcal{H}}$ and $\|\cdot\|_{\mathcal{H}}$, respectively. Let $a(\cdot, \cdot)$ be a closed sectorial sesquilinear form, densely defined in $\mathcal{H}$. "Sectorial" means that

$$
\begin{gathered}
\Re e a(v, v) \geq \alpha\|v\|_{\mathcal{H}}^{2}, \\
|\Im m a(v, v)| \leq M\left(\Re e a(v, v)-\alpha\|v\|_{\mathcal{H}}^{2}\right)
\end{gathered}
$$

for all $v \in \operatorname{Dom}(a)$ and some fixed $\alpha>0$ and $M>0$. Following the notation of Kato 9], define symmetric sesquilinear forms associated with $a$,

$$
\begin{aligned}
& \llbracket \Re e a \rrbracket(w, v)=\frac{1}{2}(a(w, v)+\overline{a(v, w)}), \\
& \llbracket \Im m a \rrbracket(w, v)=\frac{1}{2 \imath}(a(w, v)-\overline{a(v, w)}) \text {, }
\end{aligned}
$$

\footnotetext{
${ }^{1}$ Inner products and sesquilinear forms are conjugate linear in the first argument.
} 
so that $a(w, v)=\llbracket \Re e a \rrbracket(w, v)+\imath \llbracket \Im m a \rrbracket(w, v)$. Notice that (2.1) implies

$$
\alpha\|v\|_{\mathcal{H}}^{2} \leq \llbracket \Re e a \rrbracket(v, v) \leq|a(v, v)| \leq \sqrt{1+M^{2}} \llbracket \Re e a \rrbracket(v, v),
$$

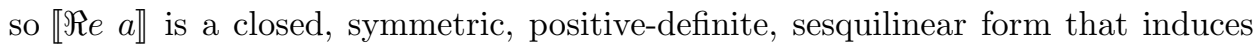
an inner product on $\operatorname{Dom}(a)$ with respect to which $\operatorname{Dom}(a)$ is a Hilbert space. $a(u, v)$ is then a bounded sesquilinear form on this Hilbert space. Furthermore, there is a closed operator, $C_{a}$, densely defined on $\mathcal{H}$ so that $\operatorname{Dom}\left(C_{a}\right)=\operatorname{Dom}(a)$ and $\llbracket \Re e a \rrbracket(v, v)=\left\|C_{a} v\right\|_{\mathcal{H}}^{2}$ (cf. 9], p. 331).

Suppose now that $\mathcal{V}=\operatorname{Dom}(a)$ is equipped with an inner product $\langle\cdot, \cdot\rangle_{\mathcal{V}}$ equivalent to $\llbracket$ Re $a \rrbracket$. The Hilbert space $\mathcal{V}$ is continuously and densely imbedded in $\mathcal{H}$ and we may assume without loss of generality that $\|u\|_{\mathcal{H}} \leq\|u\|_{\mathcal{V}}$ for all $u \in \mathcal{V}$.

Observe that any $\mathcal{H}$-bounded linear functional on $\mathcal{H}$ may be viewed immediately as the extension of some $\mathcal{V}$-bounded linear functional on $\mathcal{V}$, so letting $\mathcal{V}^{\prime}$ denote the dual space of $\mathcal{V}$, the imbedding $\mathcal{V} \hookrightarrow \mathcal{H}$ may be extended to a Gelfand triple (see e.g., 13) $\mathcal{V} \hookrightarrow \mathcal{H} \hookrightarrow \mathcal{V}^{\prime}$ with the norm on $\mathcal{V}^{\prime}$ defined by

$$
\|v\|_{\mathcal{V}^{\prime}}=\sup _{w \in \mathcal{V}} \frac{\left|\langle w, v\rangle_{\mathcal{H}}\right|}{\|w\|_{\mathcal{V}}}
$$

The Cauchy-Schwartz inequality yields $\|v\|_{\mathcal{V}^{\prime}} \leq\|v\|_{\mathcal{H}}$ for all $v \in \mathcal{H}$.

Under the hypotheses given, Kato's first representation theorem ([9], p. 322) guarantees the existence of a closed $m$-sectorial operator, $A$, defined on

$$
\operatorname{Dom}(A)=\left\{v \in \mathcal{V}|| a(w, v) \mid \leq m_{v}\|w\|_{\mathcal{H}} \text { for all } w \in \operatorname{Dom}(a)\right\}
$$

where $m_{v}$ is independent of $w$ but will generally depend on $v$. Then

$$
a(u, v)=\langle u, A v\rangle_{\mathcal{H}}
$$

for all $v \in \operatorname{Dom}(A)$ and $u \in \mathcal{V}$. Furthermore, there is a closed operator $B_{a}$ with $\operatorname{Dom}\left(B_{a}\right)=\operatorname{Dom}\left(C_{a}\right)=\operatorname{Dom}(a)$, so that $A$ may be decomposed as $A=B_{a}^{*} C_{a}$ (see e.g., 9] p. 337). “*” denotes the $\mathcal{H}$-adjoint.

Since $\operatorname{Dom}(A)$ is dense in $\mathcal{V}$ (with respect to the $\mathcal{V}$-norm) and

$$
|a(u, v)| \leq c\|u\|_{\mathcal{V}}\|v\|_{\mathcal{V}}
$$

we may calculate for any $v \in \operatorname{Dom}(A)$

$$
\|A v\|_{\mathcal{V}^{\prime}}=\sup _{u \in \mathcal{V}} \frac{\left|\langle u, A v\rangle_{\mathcal{H}}\right|}{\|u\|_{\mathcal{V}}} \leq c\|v\|_{\mathcal{V}}
$$

Thus $A$ may be extended by continuity to a bounded linear transformation from $\mathcal{V}$ to $\mathcal{V}^{\prime}$. To avoid adding a further notational burden on the reader, the two available interpretations of $A$, as an (unbounded) operator from $\operatorname{Dom}(A) \subset \mathcal{H} \rightarrow \mathcal{H}$ and as an operator from $\mathcal{V}$ to $\mathcal{V}^{\prime}$, will be distinguished only by the context in which they appear. "Dom $(A)$ " will always refer to the definition given in (2.3). Note that

$$
\|A v\|_{\mathcal{V}^{\prime}}=\sup _{u \in \mathcal{V}} \frac{|a(u, v)|}{\|u\|_{\mathcal{V}}} \geq \frac{|a(v, v)|}{\|v\|_{\mathcal{V}}} \geq \frac{\llbracket \Re e a \rrbracket(v, v)}{\|v\|_{\mathcal{V}}} \geq \hat{\alpha}\|v\|_{\mathcal{V}},
$$

thus $A$ (now extended to $\mathcal{V}$ ) is an isomorphism of $\mathcal{V}$ onto $\mathcal{V}^{\prime}$ with a bounded inverse, $T=A^{-1}$ mapping $\mathcal{V}^{\prime}$ back to $\mathcal{V}$. $T$ may be defined alternatively for each $v \in \mathcal{V}^{\prime}$, so that $T v \in \mathcal{V}$ solves

$$
\langle u, v\rangle_{\mathcal{H}}=a(u, T v)
$$

for all $u \in \mathcal{V}$. $A$ maps vectors in $\operatorname{Dom}(A)$ to $\mathcal{H}$, whereas $T$ maps vectors in $\mathcal{H} \subset \mathcal{V}^{\prime}$ back to $\operatorname{Dom}(A) \subset \mathcal{V}$. 
2.2. The gap. Given two closed subspaces, $\mathcal{M}$ and $\mathcal{N}$ of $\mathcal{H}$, the proximity of one to the other is measured in terms of the containment gap (or just gaæ2), which we define as

$$
\delta_{\mathcal{H}}(\mathcal{M}, \mathcal{N})=\sup _{x \in \mathcal{M}} \inf _{y \in \mathcal{N}} \frac{\|y-x\|_{\mathcal{H}}}{\|x\|_{\mathcal{H}}}=\sin \left(\Theta_{\max }(\mathcal{M}, \mathcal{N})\right)
$$

$\Theta_{\text {max }}(\mathcal{M}, \mathcal{N})$ is the largest canonical angle between $\mathcal{M}$ and a "closest" subspace $\widehat{\mathcal{N}}$ of $\mathcal{N}$ isomorphic to $\operatorname{dim} \mathcal{M}$. Notice that if $\operatorname{dim} \mathcal{N}<\operatorname{dim} \mathcal{M}$, then $\delta_{\mathcal{H}}(\mathcal{M}, \mathcal{N})=1$ and $\delta_{\mathcal{H}}(\mathcal{M}, \mathcal{N})=0$ if and only if $\mathcal{M} \subset \mathcal{N}$. If $\operatorname{dim} \mathcal{N}=\operatorname{dim} \mathcal{M}<\infty$, then $\delta_{\mathcal{H}}(\mathcal{M}, \mathcal{N})=\delta_{\mathcal{H}}(\mathcal{N}, \mathcal{M})$. Conversely, if both $\delta_{\mathcal{H}}(\mathcal{M}, \mathcal{N})<1$ and $\delta_{\mathcal{H}}(\mathcal{N}, \mathcal{M})<1$, then $\delta_{\mathcal{H}}(\mathcal{M}, \mathcal{N})=\delta_{\mathcal{H}}(\mathcal{N}, \mathcal{M})$ and $\mathcal{N}$ and $\mathcal{M}$ are isomorphic.

The gap can be expressed directly as the norm of a composition of projections, so that if $\Pi_{\mathcal{M}}$ and $\Pi_{\mathcal{N}}$ denote $\mathcal{H}$-orthogonal projections onto $\mathcal{M}$ and $\mathcal{N}$, respectively, then $\delta_{\mathcal{H}}(\mathcal{M}, \mathcal{N})=\left\|\left(I-\Pi_{\mathcal{N}}\right) \Pi_{\mathcal{M}}\right\|_{\mathcal{H}}$.

If $\mathcal{M}$ and $\mathcal{N}$ are closed subspaces of $\mathcal{V}$, we have the completely analogous definition of gap relative to $\mathcal{V}$ :

$$
\delta_{\mathcal{V}}(\mathcal{M}, \mathcal{N})=\sup _{x \in \mathcal{M}} \inf _{y \in \mathcal{N}} \frac{\|y-x\|_{\mathcal{V}}}{\|x\|_{\mathcal{V}}}
$$

If $A$ and $B$ are closed operators in $\mathcal{H}$ the gap between $A$ and $B$ is defined as the gap between their graphs, considered as subspaces of $\mathcal{H} \times \mathcal{H}$ :

$$
\delta_{\mathcal{H}}(A, B)=\sup _{x \in \operatorname{Dom}(A)} \inf _{y \in \operatorname{Dom}(B)} \frac{\|x-y\|_{\mathcal{H}}+\|A x-B y\|_{\mathcal{H}}}{\|x\|_{\mathcal{H}}+\|A x\|_{\mathcal{H}}} .
$$

2.3. The eigenvalue problem. Our focus rests on the (weakly posed) eigenvalue problem for $a$ :

$$
\begin{gathered}
\text { Find } \lambda \text { and } 0 \neq \hat{v} \in \operatorname{Dom}(a) \text { so that } \\
a(w, \hat{v})=\lambda\langle w, \hat{v}\rangle_{\mathcal{H}} \text { for all } w \in \operatorname{Dom}(a) .
\end{gathered}
$$

Note that $\{\lambda, \hat{v}\}$ is an eigenpair for (2.5) if and only if $\hat{v} \in \operatorname{Dom}(A)$ and $\{\lambda, \hat{v}\}$ is an eigenpair for the operator $A$; or equivalently when $\lambda \neq 0$, if $\left\{\lambda^{-1}, \hat{v}\right\}$ is an eigenpair for the operator $T$.

Denote the resolvent set of $A$ by

$$
\rho(A)=\{z \in \mathbb{C} \mid z-A \text { has a bounded inverse on } \mathcal{H}\}
$$

and the spectrum of $A$ by $\sigma(A)=\mathbb{C} \backslash \rho(A) . \quad \lambda$ is an isolated eigenvalue of (2.5) if there is a neighborhood of $\lambda$, call it $\Omega(\lambda)$, so that $\Omega(\lambda) \cap \sigma(A)$ contains only the point $\{\lambda\}$ (i.e., $\lambda$ is an isolated eigenvalue of the associated operator $A$ ). If $\lambda$ is an isolated nonzero eigenvalue of (2.5) then $\operatorname{Ker}[A-\lambda I]$ is the associated eigenspace. $\mathcal{U}=\bigcup_{k=1}^{\infty} \operatorname{Ker}\left[(A-\lambda I)^{k}\right]$ similarly will be the invariant subspace for (2.5) associated with $\lambda$. No compactness assumptions have been made for either $A$ or $T$, so a priori it may happen that (2.5) has no eigenvalues at all or those that it has may be embedded in essential spectrum (defined with respect to $A$ ) and not isolated. $\lambda$ has finite multiplicity $m$ if $\operatorname{dim} \mathcal{U}=m<\infty$. If $\lambda$ has finite multiplicity, then there is a finite integer, $r \leq m$ for which $\operatorname{Ker}\left[(A-\lambda I)^{r}\right]=\operatorname{Ker}\left[(A-\lambda I)^{r+1}\right]$. The smallest such integer is called the ascent of $\lambda$.

\footnotetext{
${ }^{2}$ Kato [9] defines the gap as $\max \left[\delta_{\mathcal{H}}(\mathcal{M}, \mathcal{N}), \delta_{\mathcal{H}}(\mathcal{N}, \mathcal{M})\right]$.
} 
Furthermore, if $\lambda$ is an isolated eigenvalue with finite multiplicity, then for each $k=1,2, \ldots,(A-\lambda)^{k}$ is a Fredholm operator with zero index implying nullity $(A-\lambda)^{k}=$ nullity $\left(A^{*}-\bar{\lambda}\right)^{k}$ for each $k$ (cf. 9], p. 239). In particular, $\bar{\lambda}$ is an eigenvalue of $A^{*}$ with the same multiplicity and ascent as $\lambda$. A "left eigenvector" associated with such a $\lambda \in \sigma(A)$ may be characterized variationally as

$$
\begin{gathered}
\text { Find } 0 \neq u_{*} \in \operatorname{Dom}(a) \text { so that } \\
a\left(u_{*}, v\right)=\lambda\left\langle u_{*}, v\right\rangle_{\mathcal{H}} \text { for all } v \in \operatorname{Dom}(a) .
\end{gathered}
$$

Note that $u_{*}$ is a solution for (2.6) if and only if $u_{*} \in \operatorname{Dom}\left(A^{*}\right)$ and $\left\{\bar{\lambda}, u_{*}\right\}$ is an eigenpair for the operator $A^{*}$.

Henceforth we will assume that there is an isolated eigenvalue $\lambda \neq 0$ for (2.5) having finite multiplicity $m$ with an associated maximal invariant subspace $\mathcal{U}$ for which we seek approximations. We denote with $\mathcal{U}_{*}$ the maximal invariant subspace for $A^{*}$ associated with $\bar{\lambda}$.

The spectral projection for $A$ onto $\mathcal{U}$ is defined by the Dunford integral

$$
E=\frac{1}{2 \pi \imath} \int_{\Gamma}(z-A)^{-1} d z
$$

where $\Gamma$ is a circle in $\mathbb{C}$ centered at $\lambda$ leaving the origin and all points of $\sigma(A)$ other than $\lambda$ in its exterior. The complementary $A$-invariant subspace is denoted $\mathcal{U}^{c}=\operatorname{Ran}(I-E)$.

Notice that $\mu=1 / \lambda$ will be an isolated eigenvalue of $T$ also with multiplicity $m$ and the same $m$-dimensional invariant subspace $\mathcal{U}$ as for $\lambda$. The spectral projection may be defined with respect to $T$ as

$$
E=\frac{1}{2 \pi \imath} \int_{\Sigma}(z-T)^{-1} d z
$$

where $\Sigma$ is a circle in $\mathbb{C}$ centered at $\mu$ leaving the origin and all points of $\sigma(T)$ other than $\mu$ in its exterior.

\section{The Galerkin Method}

3.1. Discretization. In order to approximate the eigenvalue $\lambda$ and its associated invariant subspace $\mathcal{U}$, we introduce two parameterized families of finite dimensional subspaces $\mathcal{S}_{1, h} \subset \mathcal{V}$ and $\mathcal{S}_{2, h} \subset \mathcal{V}$ - the trial and test subspaces, respectively. Assume that $\operatorname{dim} \mathcal{S}_{1, h}=\operatorname{dim} \mathcal{S}_{2, h} \stackrel{\text { def }}{=} N(h)$. Typically, the dimension $N(h)$ is monotone increasing as the "mesh size" parameter $h$ decreases.

The Galerkin method proceeds by solving an eigenvalue problem as in (2.5) for the form $a$ restricted to the finite dimensional space $\mathcal{S}_{2, h} \times \mathcal{S}_{1, h}$ :

$$
\begin{gathered}
\text { Find } \lambda_{h} \text { and } 0 \neq v_{h} \in \mathcal{S}_{1, h} \text { so that } \\
a\left(u, v_{h}\right)=\lambda_{h}\left\langle u, v_{h}\right\rangle_{\mathcal{H}} \\
\text { for all } u \in \mathcal{S}_{2, h} .
\end{gathered}
$$

The name is sometimes further qualified as either an orthogonal-Galerkin method or a Petrov-Galerkin method depending on whether $\mathcal{S}_{1, h}=\mathcal{S}_{2, h}$ or not. When $A$ is self-adjoint and $\mathcal{S}_{1, h}=\mathcal{S}_{2, h}$, this approach is called the Rayleigh-Ritz method.

For any given $h$, the computational realization proceeds by fixing bases for $\mathcal{S}_{1, h}$ as $\phi_{1}, \phi_{2}, \ldots, \phi_{N(h)}$, and for $\mathcal{S}_{2, h}$ as $\psi_{1}, \psi_{2}, \ldots, \psi_{N(h)}$. The problem (3.1) is 
then reduced to resolving the generalized matrix eigenvalue problem

$$
\begin{aligned}
& \quad \mathbf{A}_{h} \mathbf{y}=\lambda_{h} \mathbf{B}_{h} \mathbf{y} \\
& \text { where } \mathbf{A}_{h}=\left[a\left(\psi_{i}, \phi_{j}\right)\right] \in \mathbb{C}^{N(h) \times N(h)} \\
& \text { and } \mathbf{B}_{h}=\left[\left\langle\psi_{i}, \phi_{j}\right\rangle_{\mathcal{H}}\right] \in \mathbb{C}^{N(h) \times N(h)} .
\end{aligned}
$$

If an eigenvector $\mathbf{y}$ of (3.2) has components $\mathbf{y}^{t}=\left\{y_{1}, y_{2}, \ldots, y_{N(h)}\right\}$, then the corresponding $v_{h}$ that solves (3.1) is represented as $v_{h}=\sum_{j=1}^{N(h)} y_{j} \phi_{j}$.

For any $\tau \in \mathbb{C}$, define $T_{\tau}=T+\tau$, which may be defined variationally by analogy to (2.4) as that operator that satisfies

$$
\langle u, v\rangle_{\mathcal{H}}+\tau a(u, v)=a\left(u, T_{\tau} v\right)
$$

for all $u, v \in \mathcal{V}$. Notice that $\{\lambda, u\}$ is an eigenpair for (2.5) if and only if $\left\{\left(\lambda^{-1}+\tau\right), u\right\}$ is an eigenpair for the operator $T_{\tau}$ and more generally, $\sigma\left(T_{\tau}\right)=$ $\sigma(T)+\tau . T$ and $T_{\tau}$ have the same invariant subspaces $\mathcal{U}$ associated with each of the eigenvalues $\lambda^{-1}$ and $\lambda^{-1}+\tau$, respectively. The effect of a translation of $\sigma(T)$ by $\tau$ produces (from (3.3)) a discrete problem with translated spectrum. Instead of (3.2), we have

$$
\mathbf{A}_{h} \mathbf{y}=\hat{\lambda}_{h}\left(\mathbf{B}_{h}+\tau \mathbf{A}_{h}\right) \mathbf{y}
$$

The approximate spectra produced by (3.2) and (3.4) are related as $\hat{\lambda}_{h}^{-1}=\lambda_{h}^{-1}+\tau$ but eigenvectors and invariant subspaces are identical. Since our principal interest is in eigenvector approximations, choices for $\tau$ are immaterial, and particular choices will entail no loss of generality.

Assume that the following "discrete inf-sup" conditions are satisfied:

$$
\inf _{\substack{u \in \mathcal{S}_{2, h} \\\|u\|_{\mathcal{V}}=1}} \sup _{\substack{v \in \mathcal{S}_{1, h} \\\|v\|_{\mathcal{V}}=1}}|a(u, v)| \stackrel{\text { def }}{=} \beta(h)>0,
$$

and

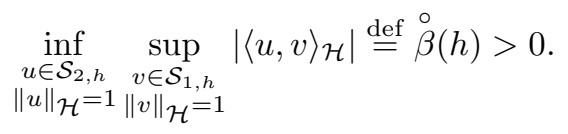

Since $\operatorname{dim} \mathcal{S}_{1, h}=\operatorname{dim} \mathcal{S}_{2, h}=N(h)$, these are equivalent to the complementary conditions,

$$
\inf _{\substack{v \in \mathcal{S}_{1, h} \\\|v\|_{\mathcal{V}}=1}} \sup _{\substack{u \in \mathcal{S}_{2, h} \\\|u\|_{\mathcal{V}}=1}}|a(u, v)|=\beta(h)>0
$$

and

$$
\inf _{\substack{v \in \mathcal{S}_{1, h} \\\|v\|_{\mathcal{H}}=1}} \sup _{\substack{u \in \mathcal{S}_{2, h} \\\|u\|_{\mathcal{H}}=1}}|\langle u, v\rangle|=\stackrel{\circ}{\beta}(h)>0,
$$

respectively. Condition (3.5) is the usual discrete inf-sup condition (cf. 11) and guarantees that $\mathbf{A}_{h}$ is invertible for each $h$. Analogously condition (3.6) guarantees that $\mathbf{B}_{h}$ is invertible for each $h$. Either (3.5) or (3.6) will guarantee that the discrete eigenvalue problem (3.2) is well posed and associated with a regular matrix pencil for each $h>0$. 
3.2. Projections. Define $P_{h}: \mathcal{V} \rightarrow \mathcal{S}_{1, h}$ as $P_{h} v=\sum_{i, j=1}^{N(h)} a\left(\psi_{j}, v\right) \gamma_{i j} \phi_{i}$ where $\left[\gamma_{i j}\right]=\mathbf{A}_{h}^{-1}$. Direct calculation verifies $P_{h}^{2}=P_{h}$, hence $P_{h}$ is a projection, albeit nonorthogonal typically. $P_{h}$ maps each $v \in \mathcal{V}$ to a unique vector, $w^{\sharp}=P_{h} v$ in $\mathcal{S}_{1, h}$ that solves

$$
\begin{gathered}
\text { Find } w^{\sharp} \in \mathcal{S}_{1, h} \text { so that } \\
a\left(u, v-w^{\sharp}\right)=0 \quad \text { for all } u \in \mathcal{S}_{2, h} .
\end{gathered}
$$

$P_{h}$ arises spontaneously in discussing solutions to boundary value problems associated with $a$. For any given $f \in \mathcal{H}$, the weakly posed boundary value problem

Find $\hat{v} \in \mathcal{V}$ so that

$$
a(w, \hat{v})=\langle w, f\rangle_{\mathcal{H}} \text { for all } w \in \mathcal{V}
$$

admits a solution $\hat{v}$ which may be approximated with a Galerkin method

Find $\hat{v}_{h} \in \mathcal{S}_{1, h}$ so that

$$
a\left(w, \hat{v}_{h}\right)=\langle w, f\rangle_{\mathcal{H}} \text { for all } w \in \mathcal{S}_{2, h}
$$

Exact and approximate solutions are related via $P_{h}$ as $\hat{v}_{h}=P_{h} \hat{v}$.

Along the same lines as above, define $P_{h}^{a}$ as $P_{h}^{a} u=\sum_{i, j=1}^{N(h)} \overline{a\left(u, \phi_{i}\right)} \bar{\gamma}_{i j} \psi_{j} . P_{h}^{a}$ is a projection onto $\mathcal{S}_{2, h}$ defined on $\mathcal{V}$ and $w^{\sharp}=P_{h}^{a} u$ solves, for any $u \in \mathcal{V}$,

$$
\begin{gathered}
\text { Find } w^{\sharp} \in \mathcal{S}_{2, h} \text { so that } \\
a\left(u-w^{\sharp}, v\right)=0 \quad \text { for all } v \in \mathcal{S}_{1, h} .
\end{gathered}
$$

Notice that (3.9) and (3.10) together imply for all $u, v \in \mathcal{V}$,

$$
a\left(u, P_{h} v\right)=a\left(P_{h}^{a} u, P_{h} v\right)=a\left(P_{h}^{a} u, v\right) .
$$

That is, $P_{h}^{a}$ is the "a-adjoint" of $P_{h}$.

Now, for all $u \in \mathcal{S}_{2, h}$ and all $v \in \mathcal{S}_{1, h}$,

$$
\begin{aligned}
\langle u, v\rangle_{\mathcal{H}} & =\left\langle P_{h}^{a} u, P_{h} v\right\rangle_{\mathcal{H}} \\
& =a\left(P_{h}^{a} u, T P_{h} v\right) \\
& =a\left(u, P_{h} T P_{h} v\right),
\end{aligned}
$$

so we have that $\lambda_{h} \neq 0$ and $v_{h}$ together solve (3.1) if and only if $\lambda_{h}^{-1}$ and $v_{h}$ constitute an eigenpair for $T_{h} \stackrel{\text { def }}{=} P_{h} T P_{h}$.

From (3.7), we find for any $v \in \mathcal{V}$ with $\left\|P_{h} v\right\|_{\mathcal{V}} \neq 0$,

$$
\begin{aligned}
0<\beta(h) & \leq \sup _{\substack{u \in \mathcal{S}_{2, h} \\
\|u\|_{\mathcal{V}}=1}} \frac{\left|a\left(u, P_{h} v\right)\right|}{\left\|P_{h} v\right\|_{\mathcal{V}}}=\sup _{\substack{u \in \mathcal{S}_{2, h} \\
\|u\|_{\mathcal{V}}=1}} \frac{|a(u, v)|}{\left\|P_{h} v\right\|_{\mathcal{V}}} \\
& \leq \sup _{\substack{u \in \mathcal{S}_{2, h} \\
\|u\|_{\mathcal{V}}=1}} \frac{c_{1}\|v\|_{\mathcal{V}}\|u\|_{\mathcal{V}}}{\left\|P_{h} v\right\|_{\mathcal{V}}} \leq c_{1} \frac{\|v\|_{\mathcal{V}}}{\left\|P_{h} v\right\|_{\mathcal{V}}}
\end{aligned}
$$

Thus,

$$
\left\|P_{h}\right\|_{\mathcal{V}} \leq c_{1} / \beta(h) .
$$

Similarly from (3.6), $\left\|P_{h}^{a}\right\|_{\mathcal{V}} \leq c_{1} / \beta(h)$. The following result leads us to conclude that both $\left\|I-P_{h}\right\|_{\mathcal{V}} \leq c_{1} / \beta(h)$ and $\left\|I-P_{h}^{a}\right\|_{\mathcal{V}} \leq c_{1} / \beta(h)$ as well. 
Lemma 3.1. If $Z$ is a bounded (nonorthogonal) projection on a Hilbert space $\mathcal{W}$ such that $Z \neq I$ and $Z \neq 0$, then $\|I-Z\|_{\mathcal{W}}=\|Z\|_{\mathcal{W}}$. Furthermore, if $\Pi$ denotes the $\mathcal{W}$-orthogonal projection onto $\operatorname{Ran}(Z)$,

$$
\frac{1}{\|Z\|_{\mathcal{W}}}\|(I-Z) u\|_{\mathcal{W}} \leq\|(I-\Pi) u\|_{\mathcal{W}} \leq\|(I-Z) u\|_{\mathcal{W}}
$$

Proof. The first assertion was proved by Kato ([8], p. 28). Since $(I-Z)=$ $(I-Z)(I-\Pi),\|(I-Z) u\|_{\mathcal{W}} \leq\|(I-Z)\|_{\mathcal{W}}\|(I-\Pi) u\|_{\mathcal{W}}$ which then gives the first inequality of (3.12). The second inequality of (3.12) is the best approximation property of orthogonal projections.

$\Pi_{1, h}$ and $\Pi_{2, h}$ will always denote orthogonal projections onto $\mathcal{S}_{1, h}$ and $\mathcal{S}_{2, h}$ respectively. However, depending on the context, they will be considered either orthogonal in $\mathcal{H}$ or orthogonal in $\mathcal{V}$ with no distinction in notation.

Define $Q_{h}: \mathcal{H} \rightarrow \mathcal{S}_{1, h}$ as $Q_{h} v=\sum_{i, j=1}^{N}\left\langle\psi_{j}, v\right\rangle_{\mathcal{H}} \stackrel{\circ}{\gamma}_{i j} \phi_{i}$ where $\left[\stackrel{\circ}{\gamma}_{i j}\right]=\mathbf{B}_{h}^{-1} . Q_{h}$ has a natural extension to $v \in \mathcal{V}^{\prime}$ so the composition of operators $A Q_{h}: \mathcal{V}^{\prime} \rightarrow \mathcal{V}^{\prime}$ and $Q_{h} A Q_{h}: \mathcal{V}^{\prime} \rightarrow \mathcal{S}_{1, h}$ are each well defined. Since $Q_{h}^{2}=Q_{h}, Q_{h}$ is also a projection, but is $\mathcal{H}$-orthogonal if and only if $\mathcal{S}_{1, h}=\mathcal{S}_{2, h} . Q_{h}$ maps each $v \in \mathcal{H}$ to a unique vector, $w^{\sharp}=Q_{h} v$ in $\mathcal{S}_{1, h}$ that solves

$$
\begin{gathered}
\text { Find } w^{\sharp} \in \mathcal{S}_{1, h} \text { so that } \\
\left\langle u, v-w^{\sharp}\right\rangle_{\mathcal{H}}=0 \quad \text { for all } u \in \mathcal{S}_{2, h} .
\end{gathered}
$$

Evidently, the $\mathcal{H}$-adjoint $Q_{h}^{*}: \mathcal{H} \rightarrow \mathcal{S}_{2, h}$ has the form $Q_{h}^{*} u=\sum_{i, j=1}^{N}\left\langle\phi_{i}, u\right\rangle_{\mathcal{H}} \overline{\stackrel{\circ}{\gamma_{i j}}} \psi_{j}$. $Q_{h}^{*}$ is a projection onto $\mathcal{S}_{2, h}$ and solves, for any $u \in \mathcal{H}$,

Find $w^{\sharp} \in \mathcal{S}_{2, h}$ so that

$$
\left\langle u-w^{\sharp}, v\right\rangle_{\mathcal{H}}=0 \quad \text { for all } v \in \mathcal{S}_{1, h} .
$$

Now, for all $u \in \mathcal{S}_{2, h}$ and all $v \in \mathcal{S}_{1, h}$,

$$
\begin{aligned}
a(u, v) & =a\left(Q_{h}^{*} u, Q_{h} v\right) \\
& =\left\langle Q_{h}^{*} u, A Q_{h} v\right\rangle_{\mathcal{H}} \\
& =\left\langle u, Q_{h} A Q_{h} v\right\rangle_{\mathcal{H}},
\end{aligned}
$$

so we have that $\lambda_{h} \neq 0$ and $v_{h}$ together solve (3.1) if and only if $\lambda_{h}$ and $v_{h}$ constitute an eigenpair for $A_{h} \stackrel{\text { def }}{=} Q_{h} A Q_{h}$.

3.3. Convergence. Convergence criteria may be framed either in $\mathcal{V}$ or in $\mathcal{H}$. Convergence criteria in $\mathcal{V}$ appear as

$$
\lim _{h \rightarrow 0} \beta(h)^{-1} \inf _{w \in \mathcal{S}_{1, h}}\|v-w\|_{\mathcal{V}}=0 \quad \text { for each } v \in \mathcal{V},
$$

$$
\lim _{h \rightarrow 0} \delta_{\mathcal{V}}\left(T_{h}, T\right)=0,
$$

Theorem 3.2 (Descloux, et al. 4, 5]). The hypotheses (3.15) and (3.16) imply:

1. Both $P_{h} \rightarrow I$ and $P_{h}^{a} \rightarrow I$ strongly in $\mathcal{V} ; P_{h}$ is uniformly $\mathcal{V}$-bounded with respect to $h$; and there is a constant $c>0$ so that

$$
\delta_{\mathcal{V}}\left(T_{h}, T\right) \leq\left\|\left(I-P_{h}\right) T P_{h}\right\|_{\mathcal{V}} \leq c \delta_{\mathcal{V}}\left(T_{h}, T\right) .
$$

2. For each compact subset, $\mathcal{R}$, of $\rho(T)$ there exists $h_{0}>0$ and $K>0$ so that $\mathcal{R} \subset \rho\left(T_{h}\right)$ and $\left\|\left(z-T_{h}\right)^{-1}\right\|_{\mathcal{V}}<K$ uniformly for $z \in \mathcal{R}$ for all $h<h_{0}$. 
3. If $\mu$ is an eigenvalue of $T$ with algebraic multiplicity $m$ and with an associated $m$-dimensional invariant subspace $\mathcal{U}$, there will be $m$ eigenvalues (counting multiplicity) of $T_{h},\left\{\mu_{h}^{1}, \mu_{h}^{2}, \ldots, \mu_{h}^{m}\right\}$ that are convergent to $\mu$ as $h \rightarrow 0$ and the associated $m$-dimensional $T_{h}$-invariant subspace $\mathcal{U}_{h}$ satisfies $\delta\left(\mathcal{U}, \mathcal{U}_{h}\right) \rightarrow 0$ as $h \rightarrow 0$.

If $\Sigma$ is a circle in $\mathbb{C}$ centered at $\mu$ leaving the origin and all points of $\sigma(T)$ other than $\mu$ in its exterior, then under the convergence assumptions (3.15) and (3.16), there will be $m$ eigenvalues of $T_{h}$, labeled as $\mu_{h}^{1}, \mu_{h}^{2}, \ldots, \mu_{h}^{m}$, that will all be contained in the interior of $\Sigma$ for sufficiently small $h$. Thus for sufficiently small $h$, the Dunford integral

$$
E_{h}=\frac{1}{2 \pi \imath} \int_{\Sigma}\left(\hat{z}-T_{h}\right)^{-1} d \hat{z}
$$

defines a spectral projection onto the $T_{h}$-invariant subspace $\mathcal{U}_{h}$ associated with $\mu_{h}^{1}, \mu_{h}^{2}, \ldots \mu_{h}^{m}$.

Analogous convergence criteria in $\mathcal{H}$ appear as

$$
\lim _{h \rightarrow 0} \stackrel{\circ}{\beta}(h)^{-1} \inf _{w \in \mathcal{S}_{1, h}}\|v-w\|_{\mathcal{H}}=0 \quad \text { for each } v \in \mathcal{H},
$$

and

$$
\lim _{h \rightarrow 0} \delta_{\mathcal{H}}\left(A_{h}, A\right)=0
$$

with similar consequences:

Theorem 3.3 (Descloux, et al. 4, 5]). The hypotheses (3.17) and (3.18) imply:

1. Both $Q_{h} \rightarrow I$ and $Q_{h}^{*} \rightarrow I$ strongly in $\mathcal{H} ; Q_{h}$ is uniformly $\mathcal{H}$-bounded with respect to $h$; and

$$
\delta_{\mathcal{H}}\left(A_{h}, A\right) \leq\left\|\left(I-Q_{h}\right) A Q_{h}\right\|_{\mathcal{H}} .
$$

If $A$ is bounded there is, in addition, a constant $c>0$ so that

$$
\left\|\left(I-Q_{h}\right) A Q_{h}\right\|_{\mathcal{H}} \leq c \delta_{\mathcal{H}}\left(A_{h}, A\right) .
$$

2. For each compact subset $\mathcal{R}$ of $\rho(A)$, there exists $h_{0}>0$ and $K>0$ so that $\mathcal{R} \subset \rho\left(A_{h}\right)$ and $\left\|\left(z-A_{h}\right)^{-1}\right\|_{\mathcal{H}}<K$ uniformly for $z \in \mathcal{R}$ for all $h<h_{0}$.

3. If $\lambda$ is an eigenvalue of $A$ with algebraic multiplicity $m$ and with an associated $m$-dimensional invariant subspace $\mathcal{U}$, there will be $m$ eigenvalues (counting multiplicity) of $A_{h},\left\{\lambda_{h}^{1}, \lambda_{h}^{2}, \ldots, \lambda_{h}^{m}\right\}$ that are convergent to $\lambda$ as $h \rightarrow 0$ and the associated $m$-dimensional $A_{h}$-invariant subspace $\mathcal{U}_{h}$ satisfies $\delta_{\mathcal{H}}\left(\mathcal{U}, \mathcal{U}_{h}\right) \rightarrow$ 0 as $h \rightarrow 0$.

If $\Gamma$ is a circle in $\mathbb{C}$ centered at $\lambda$ leaving the origin and all points of $\sigma(A)$ other than $\lambda$ in its exterior, then under the convergence assumptions (3.17) and (3.18), there will be $m$ eigenvalues of $A_{h}$, labeled as $\lambda_{h}^{1}, \lambda_{h}^{2}, \ldots, \lambda_{h}^{m}$, that will all be contained in the interior of $\Gamma$ for sufficiently small $h$. Thus for sufficiently small $h$, the Dunford integral

$$
E_{h}=\frac{1}{2 \pi \imath} \int_{\Gamma}\left(\hat{z}-A_{h}\right)^{-1} d \hat{z}
$$

defines a spectral projection for $A_{h}$ onto the invariant subspace $\mathcal{U}_{h}$ associated with $\lambda_{h}^{1}, \lambda_{h}^{2}, \ldots \lambda_{h}^{m}$. 
It will be convenient to label the complementary nonzero part of the spectrum of $T_{h}$ and $A_{h}$ as $\sigma^{c}\left(T_{h}\right)=\sigma\left(T_{h}\right) \backslash\left\{0, \mu_{h}^{1}, \mu_{h}^{2}, \ldots, \mu_{h}^{m}\right\}$ and $\sigma^{c}\left(A_{h}\right)=$ $\sigma\left(A_{h}\right) \backslash\left\{0, \lambda_{h}^{1}, \lambda_{h}^{2}, \ldots, \lambda_{h}^{m}\right\}$, respectively.

Theorem 3.4 (Babuška and Osborn [1]). Suppose $A$ is bounded and the hypotheses (3.17) and (3.18) hold. Then there is a constant $c>0$ such that

$$
\begin{aligned}
& \delta_{\mathcal{H}}\left(\mathcal{U}, \mathcal{U}_{h}\right) \leq c\left[\stackrel{\circ}{\beta}(h)^{-1} \delta_{\mathcal{H}}\left(\mathcal{U}, \mathcal{S}_{1, h}\right)\right]^{1 / r}
\end{aligned}
$$

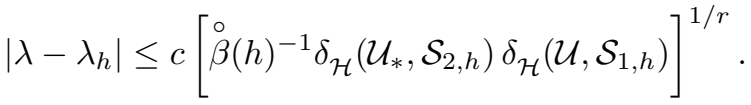

where $r$ is the ascent of the eigenvalue $\lambda$.

These appear as Theorems 8.1 and 8.3 in [1. As stated there, the proofs given in 1] presume that $A$ is compact; however, the arguments extend without change to $A$ having nontrivial essential spectrum once the convergence results of [5] come into play.

\section{Bounded $A-$ The main Results}

4.1. Basic estimates. Define

$$
\varepsilon_{\mathcal{H}}(h)=\sup _{u \in \mathcal{U}} \frac{\left\|Q_{h} A\left(I-Q_{h}\right) u\right\|_{\mathcal{H}}}{\left\|\left(I-\Pi_{1, h}\right) u\right\|_{\mathcal{H}}},
$$

where $\Pi_{1, h}$ here is the $\mathcal{H}$-orthogonal projection onto $\mathcal{S}_{1, h}$.

Theorem 4.1. Suppose the convergence hypotheses (3.17) and (3.18) hold. There exists an $h_{0}>0$ sufficiently small so that for each $h<h_{0}$ and each $u \in \mathcal{U}$ with $\|u\|_{\mathcal{H}}=1$, there is a $u_{h} \in \mathcal{U}_{h}$ so that

$$
\left\|u_{h}-Q_{h} u\right\|_{\mathcal{H}} \leq c \varepsilon_{\mathcal{H}}(h) \delta_{\mathcal{H}}\left(\mathcal{U}, \mathcal{S}_{1, h}\right)
$$

where $c>0$ is a constant independent of $h$ and independent of the choice of $u \in \mathcal{U}$.

Proof. $\operatorname{Ran}\left(E_{h}\right) \subset \mathcal{S}_{1, h}$ since $E_{h} A_{h}=A_{h} E_{h}$. Note also that $Q_{h}$ is a spectral projection for $A_{h}$ associated with all nonzero eigenvalues of $A_{h}$. Thus, $Q_{h}-E_{h}$ is a spectral projection for $A_{h}$ onto $\mathcal{U}_{h}^{c}$ associated with all nonzero eigenvalues of $A_{h}$ distinct from $\lambda$. Let $\hat{A}=\left.A\right|_{\mathcal{U}}$ denote the restriction of $A$ to $\mathcal{U}$ and Let $\hat{A}_{h}^{c}=A_{h} \mid \mathcal{U}_{h}^{c}$ denote the restriction of $A_{h}$ to $\mathcal{U}_{h}^{c}$. Then, $\hat{A}_{h}^{c}\left(Q_{h}-E_{h}\right)=\left(Q_{h}-E_{h}\right) A_{h}$ and we have

$$
\begin{aligned}
\left.\hat{A}_{h}^{c}\left(Q_{h}-E_{h}\right)\right|_{\mathcal{U}}-\left.\left(Q_{h}-E_{h}\right)\right|_{\mathcal{U}} \hat{A} \\
\quad=\left.\left(Q_{h}-E_{h}\right)\left(A_{h}-A\right)\right|_{\mathcal{U}} \\
\quad=-\left.\left(Q_{h}-E_{h}\right)\left(\left(I-Q_{h}\right) A+Q_{h} A\left(I-Q_{h}\right)\right)\right|_{\mathcal{U}} \\
\quad=-\left.\left(Q_{h}-E_{h}\right) Q_{h} A\left(I-Q_{h}\right)\right|_{\mathcal{U}} \\
\quad=-\left.\left(I-E_{h}\right) Q_{h} A\left(I-Q_{h}\right)\right|_{\mathcal{U}} .
\end{aligned}
$$

Thus, the mapping $S: \mathcal{U} \rightarrow \mathcal{U}_{h}^{c}$ given by $S=\left.\left(Q_{h}-E_{h}\right)\right|_{\mathcal{U}}$ is a solution to the Sylvester equation

$$
\hat{A}_{h}^{c} S-S \hat{A}=-\left.\left(I-E_{h}\right) Q_{h} A\left(I-Q_{h}\right)\right|_{\mathcal{U}} .
$$


There exists $K_{1}>0$ such that

$$
\left\|\left.(z-\hat{A})^{-1}\right|_{\mathcal{U}}\right\|_{\mathcal{H}} \leq\left\|(z-A)^{-1}\right\|_{\mathcal{H}} \leq K_{1}
$$

uniformly for all $z \in \Gamma$. Likewise there exists an $h_{0}>0$ and $K_{2}>0$ such that for $h<h_{0}$,

$$
\left\|\left.\left(z-\hat{A}_{h}^{c}\right)^{-1}\right|_{\mathcal{U}_{h}^{c}}\right\|_{\mathcal{H}} \leq\left\|\left(z-A_{h}\right)^{-1}\right\|_{\mathcal{H}} \leq K_{2}
$$

uniformly for $z \in \Gamma$. Therefore, the pseudospectral sets $\Lambda_{\epsilon}\left(\hat{A}_{h}\right)$ are contained in the exterior of $\Gamma$ for any $\epsilon<1 / K$ and for all $h>h_{0}$. By Lemma A.1(b), there must then be a $c_{0}>0$ independent of $h$, such that

$$
\left\|\left.\left(Q_{h}-E_{h}\right)\right|_{\mathcal{U}}\right\|_{\mathcal{U} \rightarrow \mathcal{U}_{h}^{c}} \leq c_{0}\left\|\left.\left(I-E_{h}\right) Q_{h} A\left(I-Q_{h}\right)\right|_{\mathcal{U}}\right\|_{\mathcal{U} \rightarrow \mathcal{U}_{h}^{c}} .
$$

Thus, for any $u \in \mathcal{U}$,

$$
\begin{aligned}
\left\|\left(Q_{h}-E_{h}\right) u\right\|_{\mathcal{H}} & \leq c_{0}\left\|I-E_{h}\right\|_{\mathcal{H}} \sup _{w \in \mathcal{U}} \frac{\left\|Q_{h} A\left(I-Q_{h}\right) w\right\|_{\mathcal{V}}}{\|w\|_{\mathcal{H}}} \\
& \leq c_{0}\left\|E_{h}\right\|_{\mathcal{H}} \sup _{w \in \mathcal{U}} \frac{\left\|Q_{h} A\left(I-Q_{h}\right) w\right\|_{\mathcal{V}}}{\left\|\left(I-\Pi_{1, h}\right) w\right\|_{\mathcal{H}}} \sup _{w \in \mathcal{U}} \frac{\left\|\left(I-\Pi_{1, h}\right) w\right\|_{\mathcal{V}}}{\|w\|_{\mathcal{H}}} \\
& =c_{0}\left\|E_{h}\right\|_{\mathcal{H}} \varepsilon_{\mathcal{H}}(h) \delta_{\mathcal{H}}\left(\mathcal{U}, \mathcal{S}_{1, h}\right) .
\end{aligned}
$$

Notice that since $E_{h}$ converges uniformly to $E,\left\|E_{h}\right\|_{\mathcal{H}}$ is uniformly bounded. The conclusion follows upon assigning $u_{h}=E_{h} u$.

Corollary 4.2. Suppose the convergence hypotheses (3.17) and (3.18) hold and that $\mathcal{S}_{1, h}=\mathcal{S}_{2, h}$. Then

$$
\delta_{\mathcal{H}}\left(\mathcal{U}, \mathcal{S}_{1, h}\right) \leq \delta_{\mathcal{H}}\left(\mathcal{U}, \mathcal{U}_{h}\right) \leq\left(1+c \varepsilon_{\mathcal{H}}(h)\right) \delta_{\mathcal{H}}\left(\mathcal{U}, \mathcal{S}_{1, h}\right)
$$

Proof. Note that under the hypotheses given, $Q_{h}=\Pi_{1, h}=\Pi_{2, h}=Q_{h}^{*}$. The first inequality of (4.3) follows trivially from observing that $\mathcal{U}_{h} \subset \mathcal{S}_{1, h}$. For the second, by Theorem 4.1 there exists an $h_{0}>0$ such that for each $h<h_{0}$ and $u \in \mathcal{U}$ with $\|u\|_{\mathcal{H}}=1$, there is a $\hat{u}_{h} \in \mathcal{U}_{h}$ such that $\left\|Q_{h} u-\hat{u}_{h}\right\|_{\mathcal{H}} \leq c \varepsilon_{\mathcal{H}}(h) \delta_{\mathcal{H}}\left(\mathcal{U}, \mathcal{S}_{1, h}\right)$. Then,

$$
\begin{aligned}
\min _{u_{h} \in \mathcal{U}_{h}}\left\|u-u_{h}\right\|_{\mathcal{H}} & \leq\left\|u-\hat{u}_{h}\right\|_{\mathcal{H}} \\
& \leq\left\|u-Q_{h} u\right\|_{\mathcal{H}}+\left\|Q_{h} u-\hat{u}_{h}\right\|_{\mathcal{H}} \\
& \leq \delta_{\mathcal{H}}\left(\mathcal{U}, \mathcal{S}_{1, h}\right)+c \varepsilon_{\mathcal{H}}(h) \delta_{\mathcal{H}}\left(\mathcal{U}, \mathcal{S}_{1, h}\right) .
\end{aligned}
$$

Maximizing over $u$ yields the conclusion.

Corollary 4.2 can be interpretted as saying that, provided $\varepsilon_{\mathcal{H}}(h) \rightarrow 0$, the orthogonal Galerkin method provides optimal zero order approximations to the eigenvectors of $A: \mathcal{U}_{h}$ will approach asymptotically the closest $m$-dimensional subspace in $\mathcal{S}_{1, h}$ to the exact eigenspace $\mathcal{U}$ - and this is true even if $A$ is nonselfadjoint. See the comments following Theorem 5.4

4.2. Related estimates and interpretation. Since each $Q_{h}$ is a bounded projection, $\mathcal{H}$ may be decomposed into a direct sum of complementary subspaces as $\mathcal{H}=\operatorname{Ran}\left(Q_{h}\right) \oplus \operatorname{Ker}\left(Q_{h}\right)=\mathcal{S}_{1, h} \oplus \mathcal{S}_{2, h}^{\perp}$. The operator $A$ can then be partitioned in a way that reflects this decomposition (see Figure 21). 


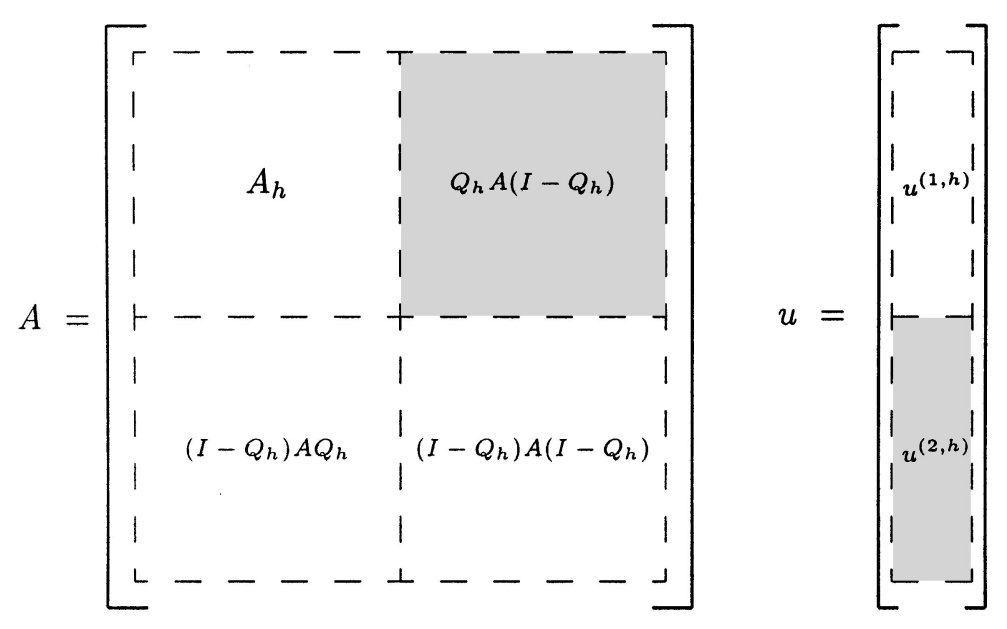

Figure 2. Partitioning $A$ and $u \in \mathcal{U}$ on $\mathcal{H}=\mathcal{S}_{1, h} \oplus \mathcal{S}_{2, h}^{\perp}$. Shading indicates influence on $\varepsilon_{\mathcal{H}}(h)$.

For each generalized eigenvector, $u \in \mathcal{U}$, define $u^{(1, h)}=Q_{h} u$ and $u^{(2, h)}=$ $\left(I-Q_{h}\right) u$. The quantity $\varepsilon_{\mathcal{H}}(h)$ is then a measure of the relative size of $Q_{h} A\left(I-Q_{h}\right)$ on $u^{(2, h)}$. This follows from observing that from Lemma 3.1

$$
\sup _{u \in \mathcal{U}} \frac{\left.\left\|Q_{h} A\left(I-Q_{h}\right) u\right\|\right)}{\left\|\left(I-Q_{h}\right) u\right\|} \leq \varepsilon_{\mathcal{H}}(h) \leq\left\|Q_{h}\right\| \sup _{u \in \mathcal{U}} \frac{\left.\left\|Q_{h} A\left(I-Q_{h}\right) u\right\|\right)}{\left\|\left(I-Q_{h}\right) u\right\|},
$$

which (provided $Q_{h} \rightarrow I$ strongly) implies immediately

$$
\sup _{u^{(2, h)} \in \mathcal{N}_{h}} \frac{\left\|Q_{h} A\left(I-Q_{h}\right) u^{(2, h)}\right\|_{\mathcal{H}}}{\left\|u^{(2, h)}\right\|_{\mathcal{H}}} \leq \varepsilon_{\mathcal{H}}(h) \leq c \sup _{u^{(2, h)} \in \mathcal{N}_{h}} \frac{\left\|Q_{h} A\left(I-Q_{h}\right) u^{(2, h)}\right\|_{\mathcal{H}}}{\left\|u^{(2, h)}\right\|_{\mathcal{H}}}
$$

for some constant $c>0$, where $\mathcal{N}_{h}=\left(I-Q_{h}\right) \mathcal{U} \subset \mathcal{S}_{2, h}^{\perp} . \mathcal{N}_{h}$ is the span of all components $u^{(2, h)}$ of (generalized) eigenvectors $u$ in $\mathcal{U}$ that lie in the direction of $\operatorname{Ker}\left(Q_{h}\right)$.

Certainly it may happen that $\varepsilon_{\mathcal{H}}(h) \nrightarrow 0$, so a variety of additional conditions will be examined in the next few sections that suffice to guarantee $\varepsilon_{\mathcal{H}}(h) \rightarrow 0$. Perhaps the simplest of these is to require that the $A_{h}^{*}$ eventually converge to $A^{*}$ in gap:

Theorem 4.3. There is a $c>0$ such that $\varepsilon_{\mathcal{H}}(h) \leq c \delta_{\mathcal{H}}\left(A_{h}^{*}, A^{*}\right)$.

Proof. Note that $I-Q_{h}=\left(I-Q_{h}\right)\left(I-\Pi_{1, h}\right)$. Thus,

$$
\begin{aligned}
\varepsilon_{\mathcal{H}}(h) & =\sup _{u \in \mathcal{U}} \frac{\left\|Q_{h} A\left(I-Q_{h}\right) u\right\|_{\mathcal{H}}}{\left\|\left(I-\Pi_{1, h}\right) u\right\|_{\mathcal{H}}} \\
& \leq\left\|Q_{h} A\left(I-Q_{h}\right)\right\|_{\mathcal{H}}=\left\|\left(I-Q_{h}^{*}\right) A^{*} Q_{h}^{*}\right\|_{\mathcal{H}} \\
& \leq c \delta_{\mathcal{H}}\left(A_{h}^{*}, A^{*}\right),
\end{aligned}
$$

using Part 1 of Theorem 3.3 .

We should not expect to do much better than the bound provided by (4.1). The bound has the "right" asymptotic behaviour in many cases and so in that sense will be best possible. 
Theorem 4.4. Suppose that $\lambda$ is a simple eigenvalue of $A$ with an associated eigenvector $u$. There exist constants $c_{0}, c_{1}>0$ (independent of $h$ ) so that for each $h$ one may find a Galerkin eigenvector $u_{h}$ with

$$
c_{0}\left\|u_{h}-Q_{h} u\right\|_{\mathcal{H}} \leq\left\|Q_{h} A\left(I-Q_{h}\right) u\right\|_{\mathcal{H}} \leq c_{1}\left\|u_{h}-Q_{h} u\right\|_{\mathcal{H}}+\left|\lambda-\lambda_{h}\right|\left\|u_{h}\right\|_{\mathcal{H}}
$$

Furthermore there exists a $c_{2}>0$ independent of $h$ so that

$$
c_{0} \frac{\left\|u_{h}-Q_{h} u\right\|_{\mathcal{H}}}{\left\|\left(I-\Pi_{1, h}\right) u\right\|_{\mathcal{H}}} \leq \varepsilon_{\mathcal{H}}(h) \leq c_{1} \frac{\left\|u_{h}-Q_{h} u\right\|_{\mathcal{H}}}{\left\|\left(I-\Pi_{1, h}\right) u\right\|_{\mathcal{H}}}+\left(c_{2} \beta(h)^{-1}\right)\left\|\left(I-\Pi_{2, h}\right) u_{*}\right\|_{\mathcal{H}},
$$

where $u_{*}$ is a "left eigenvector" satisfying (2.6).

Proof. The first inequalities in each of (4.4) and (4.5) are a consequence of Theorem 4.1. Since $\lambda$ is simple, $A u=\lambda u$ and for $h>0$ sufficiently small, $\operatorname{rank}\left(E_{h}\right)=1$ and $\lambda_{h}$ will be simple.

$$
\begin{aligned}
\left\|Q_{h} A\left(I-Q_{h}\right) u\right\|_{\mathcal{H}} & \leq\left\|\left(I-E_{h}\right) Q_{h} A\left(I-Q_{h}\right) u\right\|_{\mathcal{H}}+\left\|E_{h} Q_{h} A\left(I-Q_{h}\right) u\right\|_{\mathcal{H}} \\
& \leq\left\|\left(\hat{A}_{h}^{c} S-S \hat{A}\right) u\right\|_{\mathcal{H}}+\left\|E_{h} A u-E_{h} A_{h} u\right\|_{\mathcal{H}} \\
& \leq\left(\left\|\hat{A}_{h}^{c}\right\|_{\mathcal{H}}+|\lambda|\right)\|S u\|_{\mathcal{H}}+\left\|\lambda E_{h} u-A_{h} E_{h} u\right\|_{\mathcal{H}} \\
& \leq\left(\left\|A_{h}\right\|_{\mathcal{H}}+|\lambda|\right)\|S u\|_{\mathcal{H}}+\left\|\left(\lambda-\lambda_{h}\right) E_{h} u\right\|_{\mathcal{H}} \\
& \leq\|A\|_{\mathcal{H}}\left(1+\left\|Q_{h}\right\|_{\mathcal{H}}^{2}\right)\left\|E_{h} u-Q_{h} u\right\|_{\mathcal{H}}+\left|\lambda-\lambda_{h}\right|\left\|E_{h} u\right\|_{\mathcal{H}},
\end{aligned}
$$

where $S=\left.\left(Q_{h}-E_{h}\right)\right|_{\mathcal{U}}$ satisfies the Sylvester equation (4.2). Then (4.4) follows upon assigning $u_{h}=E_{h} u$ and observing that $Q_{h}$ is uniformly bounded in $\mathcal{H}$.

To show 4.5, note that the ascent of $\lambda$ is 1 , so from 3.20 there is a $c_{2}$ with

$$
\left|\lambda-\lambda_{h}\right| \leq c_{2} \stackrel{\circ}{\beta}_{(h)^{-1}}\left\|\left(I-\Pi_{2, h}\right) u_{*}\right\|_{\mathcal{H}}\left\|\left(I-\Pi_{h}\right) u\right\|_{\mathcal{H}}
$$

So

$$
\begin{aligned}
\varepsilon_{\mathcal{H}}(h) & =\frac{\left\|Q_{h} A\left(I-Q_{h}\right) u\right\|_{\mathcal{H}}}{\left\|\left(I-\Pi_{h}\right) u\right\|_{\mathcal{H}}} \\
& \leq c_{1} \frac{\left\|u_{h}-Q_{h} u\right\|_{\mathcal{H}}}{\left\|\left(I-\Pi_{h}\right) u\right\|_{\mathcal{H}}}+\left(c_{2} / \stackrel{\circ}{\beta}(h)^{-1}\right)\left\|\left(I-\Pi_{2, h}\right) u_{*}\right\|_{\mathcal{H}}
\end{aligned}
$$

\section{Unbounded $A$-Estimates in $\mathcal{V}$}

5.1. Basic results. The setting considered in this section is the traditional one encountered in the analysis of finite element methods. With few exceptions, much of the structure of arguments of Section 4 carry over into this setting.

Define

$$
\varepsilon_{\mathcal{V}}(h)=\sup _{u \in \mathcal{U}} \frac{\left\|P_{h} T\left(I-P_{h}\right) u\right\|_{\mathcal{V}}}{\left\|\left(I-\Pi_{1, h}\right) u\right\|_{\mathcal{V}}}
$$

where $\Pi_{1, h}$ now is the $\mathcal{V}$-orthogonal projection onto $\mathcal{S}_{1, h}$.

Theorem 5.1. Suppose the convergence hypotheses (3.15)-(3.16) hold. There exists an $h_{0}>0$ sufficiently small so that for each $h<h_{0}$ and all $u \in \mathcal{U}$, there is a $u_{h} \in \mathcal{U}_{h}$ so that

$$
\left\|u_{h}-P_{h} u\right\|_{\mathcal{V}} \leq c \varepsilon_{\mathcal{V}}(h) \delta_{\mathcal{V}}\left(\mathcal{U}, \mathcal{S}_{1, h}\right)
$$


where $c>0$ is a constant independent of $h$ and independent of the choice of $u \in \mathcal{U}$.

The proof is the same as for Theorem 4.1 formulated in the Hilbert space $\mathcal{V}$ instead of $\mathcal{H}$ and with $T, T_{h}$, and $P_{h}$ playing the roles of $A, A_{h}$, and $Q_{h}$, respectively.

Lemma 5.2. Suppose the convergence hypotheses (3.15)-(3.16) hold. If $\mathcal{V}$ is compactly imbedded in $\mathcal{H}$ (so that $T$ is compact as a mapping from $\mathcal{V}$ to itself) and if $\mathcal{S}_{2, h}$ satisfies the approximation property

$$
\lim _{h \rightarrow 0} \beta(h)^{-1} \inf _{w \in \mathcal{S}_{2, h}}\|v-w\|_{\mathcal{V}}=0 \quad \text { for each } v \in \mathcal{V},
$$

then $\varepsilon_{\mathcal{V}}(h) \rightarrow 0$ as $h \rightarrow 0$.

Proof. Since $a$ is bounded and coercive on $\mathcal{V}$, there is a bounded and invertible linear operator on $\mathcal{V}, \mathcal{A}$, such that $a(u, v)=\langle u, \mathcal{A} v\rangle_{\mathcal{V}}$. Let " $\star$ " denote the $\mathcal{V}$-adjoint and observe that $P_{h}^{\star} u=\sum_{i, j=1}^{N}\left\langle\phi_{i}, u\right\rangle_{\mathcal{V}} \overline{\gamma_{i j}} \mathcal{A}^{\star} \psi_{j}$, so that, in particular, $\operatorname{Ran}\left(P_{h}^{\star}\right)=$ $\mathcal{A}^{\star} \mathcal{S}_{2, h}$. Let $\Pi_{2, h}$ denote the $\mathcal{V}$-orthogonal projection onto $\mathcal{S}_{2, h}$ and notice that $\mathcal{A}^{\star} \Pi_{2, h} \mathcal{A}^{-\star}$ is a projection (no longer orthogonal, in general) onto $\operatorname{Ran}\left(P_{h}^{\star}\right)$. Then for any $u \in \mathcal{V},(5.2)$ implies

$$
\begin{aligned}
\left\|\left(I-P_{h}^{\star}\right) u\right\|_{\mathcal{V}} & =\left\|\left(I-P_{h}^{\star}\right)\left(I-\mathcal{A}^{\star} \Pi_{2, h} \mathcal{A}^{-\star}\right) u\right\|_{\mathcal{V}} \\
& =\left\|\left(I-P_{h}^{\star}\right) \mathcal{A}^{\star}\left(I-\Pi_{2, h}\right) \mathcal{A}^{-\star} u\right\|_{\mathcal{V}} \\
& \leq\left\|I-P_{h}^{\star}\right\|_{\mathcal{V}}\left\|\mathcal{A}^{\star}\right\|_{\mathcal{V}}\left\|\left(I-\Pi_{2, h}\right) \mathcal{A}^{-\star} u\right\|_{\mathcal{V}} \\
& \leq \frac{c}{\beta(h)}\left\|\left(I-\Pi_{2, h}\right) \mathcal{A}^{-\star} u\right\|_{\mathcal{V}} \\
& =\frac{c}{\beta(h)} \inf _{w \in \mathcal{S}_{2, h}}\left\|\mathcal{A}^{-\star} u-w\right\|_{\mathcal{V}} \rightarrow 0
\end{aligned}
$$

for some constant $c$. Thus, $P_{h}^{\star}$ converges strongly to $I$ in $\mathcal{V}$. Since $T^{\star}$ is compact, $\left\|\left(I-P_{h}^{\star}\right) T^{\star}\right\|_{\mathcal{V}} \rightarrow 0$ and

$$
\begin{aligned}
\varepsilon_{\mathcal{V}}(h) & =\sup _{u \in \mathcal{U}} \frac{\left\|P_{h} T\left(I-P_{h}\right)\left(I-\Pi_{1, h}\right) u\right\|_{\mathcal{V}}}{\left\|\left(I-\Pi_{1, h}\right) u\right\|_{\mathcal{V}}} \\
& \leq\left\|P_{h} T\left(I-P_{h}\right)\right\|_{\mathcal{V}} \\
& =\left\|\left(I-P_{h}^{\star}\right) T^{\star} P_{h}^{\star}\right\|_{\mathcal{V}} \\
& \leq\left\|\left(I-P_{h}^{\star}\right) T^{\star}\right\|_{\mathcal{V}}\left\|P_{h}^{\star}\right\|_{\mathcal{V}} \rightarrow 0 .
\end{aligned}
$$

Even when $T$ is not compact, additional conditions on $\mathcal{S}_{2, h}$ can yield the same result:

Lemma 5.3. Let $T^{*}$ denote the $\mathcal{H}$-adjoint of $T$. Suppose the convergence hypotheses (3.15)-(3.16) hold. If $\mathcal{S}_{2, h}$ satisfies the approximation properties

$$
\lim _{h \rightarrow 0} \beta(h)^{-1} \inf _{w \in \mathcal{S}_{2, h}}\|v-w\|_{\mathcal{V}}=0 \quad \text { for each } v \in \mathcal{V},
$$

and

$$
\sup _{\substack{v \in \mathcal{S}_{2, h} \\\|v\|_{\mathcal{V}}=1}} \inf _{w \in \mathcal{S}_{2, h}}\left\|T^{*} v-w\right\|_{\mathcal{V}} \stackrel{\text { def }}{=} \gamma(h) \rightarrow 0 \quad \text { as } h \rightarrow 0 .
$$

Then $\varepsilon_{\mathcal{V}}(h) \rightarrow 0$ as $h \rightarrow 0$ and $\varepsilon_{\mathcal{V}}(h)=\mathcal{O}(\gamma(h))$. 
Proof. We first verify that $T^{*}$ maps $\mathcal{V}$ to $\mathcal{V}$. For any $u \in \mathcal{H}, v \in \operatorname{Dom}(a)$, $\left|\langle u, v\rangle_{\mathcal{H}}\right| \leq \frac{1}{\alpha}\|u\|_{\mathcal{H}}\left\|C_{a} v\right\|_{\mathcal{H}}$. (Recall $B_{a}$ and $C_{a}$ were defined in Section 2, Thus, for $u \in \operatorname{Dom}(a), w \in \operatorname{Ran}\left(\left.C_{a}\right|_{\operatorname{Dom}(A)}\right.$ ), (so that $w=C_{a} v$ for some $v \in \operatorname{Dom}(A)$ ), one may observe

$$
\begin{aligned}
\left|\left\langle T^{*} u, B_{a}^{*} w\right\rangle_{\mathcal{H}}\right| & =\left|\left\langle T^{*} u, B_{a}^{*} C_{a} v\right\rangle_{\mathcal{H}}\right| \\
& =\left|\left\langle T^{*} u, A v\right\rangle_{\mathcal{H}}\right| \\
& =\left|\langle u, v\rangle_{\mathcal{H}}\right| \\
& \leq\left(\frac{\|u\|_{\mathcal{H}}}{\alpha}\right)\|w\|_{\mathcal{H}} .
\end{aligned}
$$

Since $\operatorname{Dom}(A)$ is a core for $C_{a}, \operatorname{Ran}\left(\left.C_{a}\right|_{\operatorname{Dom}(A)}\right)$ is dense in $\mathcal{H}$ and as a consequence $T^{*} u \in \operatorname{Dom}\left(B_{a}^{* *}\right)=\operatorname{Dom}\left(B_{a}\right)=\operatorname{Dom}(a)$. For $u \in \operatorname{Dom}(a), v \in \operatorname{Dom}(A)$, then

$$
\begin{aligned}
a\left(T^{a} u, v\right)=a(u, T v) & =\langle u, v\rangle_{\mathcal{H}} \\
& =\langle u, T A v\rangle_{\mathcal{H}} \\
& =\left\langle T^{*} u, A v\right\rangle_{\mathcal{H}}=a\left(T^{*} u, v\right)
\end{aligned}
$$

(the last equality being a consequence of $T^{*} u \in \operatorname{Dom}(a)$ ), and so, $T^{a}=T^{*}$.

Since $\|\cdot\|_{\mathcal{V}}$ and $\llbracket \Re e a \rrbracket$ are equivalent norms on $\mathcal{V}$, there is an $m>0$ so that $m\|u\|_{\mathcal{V}}^{2} \leq|a(u, u)|$ and

$$
\begin{aligned}
m\left\|P_{h} T\left(I-P_{h}\right) v\right\|_{\mathcal{V}} & \leq \frac{\left|a\left(P_{h} T\left(I-P_{h}\right) v, P_{h} T\left(I-P_{h}\right) v\right)\right|}{\left\|P_{h} T\left(I-P_{h}\right) v\right\|_{\mathcal{V}}} \\
& \leq \sup _{\substack{u \in \mathcal{V} \\
\|u\|_{\mathcal{V}}=1}}\left|a\left(u, P_{h} T\left(I-P_{h}\right) v\right)\right| \\
& =\sup _{\substack{u \in \mathcal{V} \\
\|u\|_{\mathcal{V}}=1}}\left|a\left(\left(I-P_{h}^{a}\right) T^{*} P_{h}^{a} u, v\right)\right| \\
& \leq \sup _{\substack{u \in \mathcal{V} \\
\|u\|_{\mathcal{V}}=1}} c_{1}\|v\|_{\mathcal{V}}\left\|\left(I-P_{h}^{a}\right) T^{*} P_{h}^{a} u\right\|_{\mathcal{V}} \\
& =c_{1}\|v\|_{\mathcal{V}}\left\|\left(I-P_{h}^{a}\right) T^{*} P_{h}^{a}\right\|_{\mathcal{V}} .
\end{aligned}
$$

Thus, $\varepsilon_{\mathcal{V}}(h) \leq\left\|P_{h} T\left(I-P_{h}\right)\right\|_{\mathcal{V}} \leq\left(c_{1} / m\right)\left\|\left(I-P_{h}^{a}\right) T^{*} P_{h}^{a}\right\|_{\mathcal{V}}$. Now, notice that $\operatorname{Ran}\left(P_{h}^{a}\right)=\mathcal{S}_{2, h}$, so $I-P_{h}^{a}=\left(I-P_{h}^{a}\right)\left(I-\Pi_{2, h}\right)$, where $\Pi_{2, h}$ is the $\mathcal{V}$-orthogonal projection onto $\mathcal{S}_{2, h}$. Now,

$$
\begin{aligned}
\left\|\left(I-P_{h}^{a}\right) T^{*} P_{h}^{a}\right\|_{\mathcal{V}} & \leq\left\|\left(I-P_{h}^{a}\right)\left(I-\Pi_{2, h}\right) T^{*} \Pi_{2, h} P_{h}^{a}\right\|_{\mathcal{V}} \\
& \leq\left\|\left(I-P_{h}^{a}\right)\right\|_{\mathcal{V}}\left\|P_{h}^{a}\right\|_{\mathcal{V}}\left\|\left(I-\Pi_{2, h}\right) T^{*} \Pi_{2, h}\right\|_{\mathcal{V}} \\
& =\left\|\left(I-P_{h}^{a}\right)\right\|_{\mathcal{V}}\left\|P_{h}^{a}\right\|_{\mathcal{V}} \sup _{\substack{v \in \mathcal{S}_{2, h} \\
\|v\|_{\mathcal{V}}=1}} \inf _{w \in \mathcal{S}_{2, h}}\left\|T^{*} v-w\right\|_{\mathcal{V}} \\
& \leq c \gamma(h) \rightarrow 0 .
\end{aligned}
$$

If $a$ is symmetric (so that $\llbracket \Re e a \rrbracket=a$ and $\llbracket \Im m a \rrbracket=0$ ) and if $a(\cdot, \cdot)$ itself is used as the inner product for $\mathcal{V}$, then $T$ is a self-adjoint operator in $\mathcal{V}$. If additionally $\mathcal{S}_{1, h}=\mathcal{S}_{2, h}$, then $P_{h}$ is a $\mathcal{V}$-orthogonal projection and $T_{h}$ is then also self-adjoint. In this circumstance, $u_{h}$ is asymptotically the closest vector out of $\mathcal{S}_{1, h}$ to $u$ (with respect to the $a$-norm on $\mathcal{V}$ ): 
Theorem 5.4. Suppose $a$ is symmetric, $a(u, v)=\langle u, v\rangle_{\mathcal{V}}$, and that $\mathcal{S}_{1, h}=\mathcal{S}_{2, h}$. Then

$$
\varepsilon_{\mathcal{V}}(h) \leq c_{1} \delta_{\mathcal{V}}\left(T_{h}, T\right)
$$

and

$$
1 \leq \frac{\delta_{\mathcal{V}}\left(\mathcal{U}, \mathcal{U}_{h}\right)}{\delta_{\mathcal{V}}\left(\mathcal{U}, \mathcal{S}_{1, h}\right)} \leq 1+c_{2} \delta_{\mathcal{V}}\left(T_{h}, T\right) .
$$

Proof. Note that under the hypotheses given, $P_{h}=\Pi_{1, h}=\Pi_{2, h}=P_{h}^{a}$. Thus,

$$
\begin{aligned}
\varepsilon_{\mathcal{V}}(h) & =\sup _{u \in \mathcal{U}} \frac{\left\|\Pi_{1, h} T\left(I-\Pi_{1, h}\right) u\right\|_{\mathcal{V}}}{\left\|\left(I-\Pi_{1, h}\right) u\right\|_{\mathcal{V}}} \\
& \leq\left\|\Pi_{1, h} T\left(I-\Pi_{1, h}\right)\right\|_{\mathcal{V}} \\
& =\left\|\left(I-\Pi_{1, h}\right) T \Pi_{1, h}\right\|_{\mathcal{V}} \\
& \leq c_{1} \delta_{\mathcal{V}}\left(T_{h}, T\right) .
\end{aligned}
$$

The first inequality of (5.7) follows trivially from observing that $\mathcal{U}_{h} \subset \mathcal{S}_{1, h}$. For the second, by Theorem 5.1 there exists an $h_{0}>0$ such that for each $h<h_{0}$ and $u \in \mathcal{U}$ with $\|u\|_{\mathcal{V}}=1$, there is a $\hat{u}_{h} \in \mathcal{U}_{h}$ such that $\left\|P_{h} u-\hat{u}_{h}\right\|_{\mathcal{V}} \leq c \varepsilon_{\mathcal{V}}(h) \delta_{\mathcal{V}}\left(\mathcal{U}, \mathcal{S}_{1, h}\right)$. Then,

$$
\begin{aligned}
\min _{u_{h} \in \mathcal{U}_{h}}\left\|u-u_{h}\right\|_{\mathcal{V}} & \leq\left\|u-\hat{u}_{h}\right\|_{\mathcal{V}} \\
& \leq\left\|u-P_{h} u\right\|_{\mathcal{V}}+\left\|P_{h} u-\hat{u}_{h}\right\|_{\mathcal{V}} \\
& \leq \delta_{\mathcal{V}}\left(\mathcal{U}, \mathcal{S}_{1, h}\right)+c \varepsilon_{\mathcal{V}}(h) \delta_{\mathcal{V}}\left(\mathcal{U}, \mathcal{S}_{1, h}\right) .
\end{aligned}
$$

Maximizing over $u$ yields the conclusion.

Theorem [5.4 was essentially given by Chatelin [3], refined by Babuška and Osborn [1] - each for compact self-adjoint $T$. Recently, a more general result of this sort allowing for noncompact self-adjoint $T$ was given by Knyazev [10].

5.2. Elliptic boundary value problems: Finite elements. Let $\Omega$ be a bounded open subset of $\mathbb{R}^{n}$ with a boundary $\partial \Omega$ that is at least $C^{r+1,1}$ for some integer $r>0$. Given real coefficient functions $a_{i j}, b_{i}, c \in C^{r}(\bar{\Omega})$, consider the second order elliptic differential operator $A$ defined by

$$
A(\mathbf{x}, D) v=-\sum_{i, j=1}^{n} D_{j} a_{i j}(\mathbf{x}) D_{i} v+\sum_{i=1}^{n} b_{i}(\mathbf{x}) D_{i} v+c(\mathbf{x}) v \text { in } \Omega
$$

with $v=0$ on $\partial \Omega$, and the related adjoint operator given by

$$
A^{*}(\mathbf{x}, D) u=-\sum_{i, j=1}^{n} D_{i} a_{i j}(\mathbf{x}) D_{j} u-\sum_{i=1}^{n} D_{i}\left(b_{i}(\mathbf{x}) u\right)+c(\mathbf{x}) u \quad \text { in } \Omega
$$

with $u=0$ on $\partial \Omega$.

Suppose that $A(\mathbf{x}, D)$ is uniformly strongly elliptic. The associated bilinear form

$$
\begin{aligned}
a(w, v)= & \sum_{i, j=1}^{n} \int_{\Omega} a_{i j}(\mathbf{x})\left[D_{j} w\right]\left[D_{i} v\right] d \mathbf{x}+\sum_{j=1}^{n} \int_{\Omega} w b_{j}(\mathbf{x})\left[D_{j} v\right] d \mathbf{x} \\
& +\int_{\Omega} c(\mathbf{x}) w v d \mathbf{x},
\end{aligned}
$$


defined on $\operatorname{Dom}(a)=H_{0}^{1}(\Omega)$ is a closed sectorial bilinear form densely defined in $\mathcal{H}=L^{2}(\Omega)$ and $A$ is manifested as a densely defined $m$-sectorial operator on $\mathcal{H}$ which can be extended to a continuous bijection from $\mathcal{V}=H_{0}^{1}(\Omega)$ onto the dual space $\mathcal{V}^{\prime}=H^{-1}(\Omega)$. Here and elsewhere, $H^{p}(\Omega)$ denotes the completion of the vector space $C^{\infty}$ with respect to the norm

$$
\|u\|_{H^{p}(\Omega)}=\sum_{|\alpha| \leq p} \int_{\Omega}\left|D^{\alpha} v\right|^{2} d \mathbf{x} .
$$

The associated seminorm is defined as

$$
|u|_{H^{p}(\Omega)}=\sum_{|\alpha|=p} \int_{\Omega}\left|D^{\alpha} v\right|^{2} d \mathbf{x} .
$$

Results governing regularity of solutions to elliptic problems (e.g., [13] p. 328) guarantee, for any $f \in H^{r-1}(\Omega)$, the weakly posed problem

$$
a(w, v)=\langle w, f\rangle_{L^{2}(\Omega)} \quad \text { for all } w \in H_{0}^{1}(\Omega)
$$

has a solution $v \in H^{r+1}(\Omega) \cap H_{0}^{1}(\Omega)$. Since $T$ is a closed mapping from $H^{r-1}(\Omega)$ to $H^{r+1}(\Omega)$, there exists a constant $c>0$ such that $\|T f\|_{H^{r+1}(\Omega)} \leq c\|f\|_{H^{r-1}(\Omega)}$ for all $f \in H^{r-1}(\Omega)$. Furthermore, if $\mathcal{U}$ denotes an invariant subspace of $A$ associated with an isolated eigenvalue of $A$ with finite multiplicity then $\mathcal{U} \subset H^{r+1}(\Omega)$.

Likewise the adjoint problem,

$$
a(u, w)=\langle w, g\rangle_{L^{2}(\Omega)} \quad \text { for all } w \in H^{1}(\Omega)
$$

has a solution $u \in H^{r+1}(\Omega)$ for any $g \in H^{r-1}(\Omega)$. So, in particular, if $g \in \mathcal{V} \subset$ $H^{1}(\Omega)$, then $T^{*} g=\left(A^{*}\right)^{-1} g \in H^{3}(\Omega)$.

Apply the Galerkin method with $\mathcal{S}_{h}=\mathcal{S}_{1, h}=\mathcal{S}_{2, h}$ chosen to be a family of finite dimensional subspaces of $\mathcal{V}$, so that for all integers $0<k \leq r$ and some fixed $c>0$, $u \in H^{r+1}(\Omega)$ implies

$$
\inf _{v \in \mathcal{S}_{h}}\|u-v\|_{H^{1}(\Omega)} \leq c h^{k}|u|_{H^{k+1}(\Omega)}
$$

For example, $C^{0}$-finite element spaces constructed from piecewise polynomials of degree at least $r$ would satisfy this condition.

The discrete inf-sup condition (3.5) is satisfied with $\beta(h)=\alpha>0$. Thus, the convergence condition (3.15) is immediately satisfied. It remains to verify that (3.16) holds. Note that for every $x \in \mathcal{S}_{h}=\operatorname{Dom}\left(T_{h}\right), T x \in H^{3}(\Omega)$ and

$$
\begin{aligned}
\inf _{y \in H^{1}(\Omega)} \frac{\|x-y\|_{H^{1}(\Omega)}+\left\|T_{h} x-T y\right\|_{H^{1}(\Omega)}}{\|x\|_{H^{1}(\Omega)}+\left\|T_{h} x\right\|_{H^{1}(\Omega)}} & \leq \frac{\left\|T_{h} x-T x\right\|_{H^{1}(\Omega)}}{\|x\|_{H^{1}(\Omega)}+\left\|T_{h} x\right\|_{H^{1}(\Omega)}} \\
& =\frac{\|(I-P h) T x\|_{H^{1}(\Omega)}}{\|x\|_{H^{1}(\Omega)}+\left\|T_{h} x\right\|_{H^{1}(\Omega)}} \\
& \leq \frac{c h^{2}|T x|_{H^{3}(\Omega)}}{\|x\|_{H^{1}(\Omega)}+\left\|T_{h} x\right\|_{H^{1}(\Omega)}} \\
& \leq \frac{c h^{2}\|x\|_{H^{1}(\Omega)}}{\|x\|_{H^{1}(\Omega)}+\left\|T_{h} x\right\|_{H^{1}(\Omega)}} \\
& \leq c h^{2} .
\end{aligned}
$$

Thus, $\lim _{h \rightarrow 0} \delta_{\mathcal{V}}\left(T_{h}, T\right)=0$. 
Although Theorem 5.2 is applicable, Theorem 5.3 will yield a concrete rate once we estimate

$$
\gamma(h)=\sup _{\substack{v \in \mathcal{S}_{h} \\\|v\|_{\mathcal{V}}=1}} \inf _{w \in \mathcal{S}_{h}}\left\|T^{*} v-w\right\|_{\mathcal{V}}
$$

For any $v \in \mathcal{S}_{h}, T^{*} v \in H^{3}(\Omega)$ and so

$$
\begin{aligned}
\inf _{w \in \mathcal{S}_{h}}\left\|T^{*} v-w\right\|_{\mathcal{V}} & =\left\|\left(I-\Pi_{h}\right) T^{*} v\right\|_{\mathcal{V}} \\
& \leq \operatorname{ch}^{2}\left|T^{*} v\right|_{H^{3}(\Omega)} \\
& \leq \operatorname{ch}^{2}\|v\|_{H^{1}(\Omega)} .
\end{aligned}
$$

Thus, $\gamma(h) \leq c h^{2}$.

Since $\mathcal{U} \subset H^{r+1}(\Omega)$,

$$
\begin{aligned}
\delta_{\mathcal{V}}\left(\mathcal{U}, \mathcal{S}_{h}\right) & =\sup _{x \in \mathcal{U}} \inf _{y \in \mathcal{S}_{h}} \frac{\|y-x\|_{\mathcal{V}}}{\|x\|_{\mathcal{V}}}=\frac{\left\|\left(I-\Pi_{h}\right) x\right\|_{\mathcal{V}}}{\|x\|_{\mathcal{V}}} \\
& \leq \operatorname{ch}^{r} \frac{\mid x \mathbf{|}_{H^{r+1}}(\Omega)}{\|x\|_{\mathcal{V}}} \leq \operatorname{ch}^{r} .
\end{aligned}
$$

Theorem 5.1 asserts that there exists an $h_{0}>0$ sufficiently small so that for each $h<h_{0}$ and all $u \in \mathcal{U}$, there is a $u_{h} \in \mathcal{U}_{h}$ so that

$$
\left\|u_{h}-P_{h} u\right\|_{\mathcal{V}} \leq c h^{r+2}
$$

whereas $\left\|u_{h}-u\right\|_{\mathcal{V}}$ and $\left\|u-P_{h} u\right\|_{\mathcal{V}}$ will each be only of order $h^{r}$ typically.

\section{Unbounded $A$-Estimates in $\mathcal{H}$}

6.1. Basic results. In the $\mathcal{V}$-setting explored in Section 苟, orthogonality of $P_{h}$ and the related best approximation property in $\mathcal{V}$ could be developed only for self-adjoint $A$. In contrast, estimates in $\mathcal{H}$ such as were found in Section 4 have particular appeal since whenever $\mathcal{S}_{1, h}=\mathcal{S}_{2, h}, Q_{h}$ will be an orthogonal projection in $\mathcal{H}$, notwithstanding asymmetry in $a$ and nonselfadjointness of $A$. Unfortunately, those estimates obtained in Section 4 depend fundamentally on the boundedness of $A$. In particular, if $A$ is unbounded then $\varepsilon_{\mathcal{H}}(h)$ might not be uniformly bounded in $h$, much less go to zero.

We define an expression that plays a role analogous to that of $\varepsilon_{\mathcal{H}}(h)$ in Section 田

$$
\stackrel{\circ}{\mathcal{H}}_{\mathcal{H}}(h)=\sup _{u \in \mathcal{U}} \frac{\left\|\left(P_{h}-Q_{h}\right) u\right\|_{\mathcal{H}}}{\left\|\left(I-\Pi_{1, h}\right) u\right\|_{\mathcal{H}}}
$$

where $\Pi_{1, h}$ here is once again the $\mathcal{H}$-orthogonal projection onto $\mathcal{S}_{1, h}$.

Theorem 6.1. Suppose the convergence hypotheses (3.17) and (3.18) hold. There exists an $h_{0}>0$ sufficiently small so that for each $h<h_{0}$ and all $u \in \mathcal{U}$, there is a $u_{h} \in \mathcal{U}_{h}$ so that

$$
\left\|u_{h}-Q_{h} u\right\|_{\mathcal{H}} \leq c \stackrel{\circ}{\varepsilon}_{\mathcal{H}}(h) \delta_{\mathcal{H}}\left(\mathcal{U}, \mathcal{S}_{1, h}\right),
$$

where $c>0$ is a constant independent of $h$ and independent of the choice of $u \in \mathcal{U}$. 
Proof. Since $E_{h} A_{h}=A_{h} E_{h}, \operatorname{Ran}\left(E_{h}\right) \subset \mathcal{S}_{1, h}$ Note also that $Q_{h}$ is a spectral projection for $A_{h}$ associated with all nonzero eigenvalues of $A_{h}$. Thus, $Q_{h}-E_{h}$ is a spectral projection for $A_{h}$ onto $\mathcal{U}_{h}^{c}$. Let $\hat{A}=\left.A\right|_{\mathcal{U}}$ denote the restriction of $A$ to $\mathcal{U}$ and Let $\hat{A}_{h}^{c}=\left.A_{h}\right|_{\mathcal{U}_{h}^{c}}$ denote the restriction of $A_{h}$ to $\mathcal{U}_{h}^{c}$. Then, $\hat{A}_{h}^{c}\left(Q_{h}-E_{h}\right)=\left(Q_{h}-E_{h}\right) A_{h}$ and we have

$$
\begin{aligned}
\left.\hat{A}_{h}^{c}\left(Q_{h}-E_{h}\right)\right|_{\mathcal{U}}-\left.\left(Q_{h}-E_{h}\right)\right|_{\mathcal{U}} \hat{A} & =\left.\left(Q_{h}-E_{h}\right)\left(A_{h}-A\right)\right|_{\mathcal{U}} \\
& =-\left.\left(Q_{h}-E_{h}\right)\left(\left(I-Q_{h}\right) A+Q_{h} A\left(I-Q_{h}\right)\right)\right|_{\mathcal{U}} \\
& =-\left.\left(Q_{h}-E_{h}\right) Q_{h} A\left(I-Q_{h}\right)\right|_{\mathcal{U}} \\
& =-\left.\left(I-E_{h}\right) Q_{h} A\left(I-Q_{h}\right)\right|_{\mathcal{U}} .
\end{aligned}
$$

Now premultiply by $T_{h}$ and postmultiply by $T$ to find

$$
\begin{aligned}
\left.\left(Q_{h}-E_{h}\right)\right|_{\mathcal{U}} \hat{T}-\left.\hat{T}_{h}^{c}\left(Q_{h}-E_{h}\right)\right|_{\mathcal{U}} & =-\left.T_{h}\left(I-E_{h}\right) Q_{h} A\left(I-Q_{h}\right) T\right|_{\mathcal{U}} \\
& =-\left.\left(I-E_{h}\right) T_{h} Q_{h} A\left(I-Q_{h}\right) T\right|_{\mathcal{U}} \\
& =-\left.\left(I-E_{h}\right)\left(T_{h} Q_{h} A T-T_{h} Q_{h} A Q_{h} T\right)\right|_{\mathcal{U}} \\
& =-\left.\left(I-E_{h}\right)\left(T_{h} Q_{h}-Q_{h} T\right)\right|_{\mathcal{U}} \\
& =-\left.\left(I-E_{h}\right)\left(P_{h} T-Q_{h} T\right)\right|_{\mathcal{U}} .
\end{aligned}
$$

Thus, the mapping $S: \mathcal{U} \rightarrow \mathcal{U}_{h}^{c}$ given by $S=\left.\left(Q_{h}-E_{h}\right)\right|_{\mathcal{U}}$ is a solution to the Sylvester equation

$$
\hat{T}_{h}^{c} S-S \hat{T}=\left.\left(I-E_{h}\right)\left(P_{h}-Q_{h}\right) T\right|_{\mathcal{U}} .
$$

The goal now is to show that the bounds developed in the Appendix are applicable. There exists a $K_{1}>0$ such that

$$
\left\|\left.(z-\hat{A})^{-1}\right|_{\mathcal{U}}\right\|_{\mathcal{H}} \leq\left\|(z-A)^{-1}\right\|_{\mathcal{H}} \leq K_{1}
$$

uniformly for all $z \in \Gamma$. Likewise there exists an $h_{0}>0$ and $K_{2}>0$ such that for $h<h_{0}$,

$$
\left\|\left(z-\hat{A}_{h}^{c}\right)^{-1} \mid \mathcal{U}_{h}^{c}\right\|_{\mathcal{H}} \leq\left\|\left(z-A_{h}\right)^{-1}\right\|_{\mathcal{H}} \leq K_{2}
$$

uniformly for $z \in \Gamma$. Therefore, the pseudospectral sets $\Lambda_{\epsilon}\left(\hat{A}_{h}\right)$ are contained in the exterior of $\Gamma$ for any $\epsilon<1 / K_{2}$ and for all $h>h_{0}$. By Lemma A.1(b), there must then be a $c>0$ independent of $h$, such that

$$
\left\|\left.\left(Q_{h}-E_{h}\right)\right|_{\mathcal{U}}\right\|_{\mathcal{U} \rightarrow \mathcal{U}_{h}^{c}} \leq c\left\|\left.\left(I-E_{h}\right) Q_{h} A\left(I-Q_{h}\right)\right|_{\mathcal{U}}\right\|_{\mathcal{U} \rightarrow \mathcal{U}_{h}^{c}} .
$$

Thus, for any $u \in \mathcal{U}$,

$$
\begin{aligned}
\left\|\left(Q_{h}-E_{h}\right) u\right\|_{\mathcal{H}} & \leq c\left\|I-E_{h}\right\|_{\mathcal{H}} \sup _{w \in \mathcal{U}} \frac{\left\|Q_{h} A\left(I-Q_{h}\right) w\right\|_{\mathcal{H}}}{\|w\|_{\mathcal{H}}} \\
& \leq c\left\|E_{h}\right\|_{\mathcal{H}} \sup _{w \in \mathcal{U}} \frac{\left\|Q_{h} A\left(I-Q_{h}\right) w\right\|_{\mathcal{H}}}{\left\|\left(I-\Pi_{1, h}\right) w\right\|_{\mathcal{H}}} \sup _{w \in \mathcal{U}} \frac{\left\|\left(I-\Pi_{1, h}\right) w\right\|_{\mathcal{V}}}{\|w\|_{\mathcal{H}}} \\
& =c\left\|E_{h}\right\|_{\mathcal{H}} \stackrel{\circ}{\mathcal{H}}_{\mathcal{H}}(h) \delta_{\mathcal{H}}\left(\mathcal{U}, \mathcal{S}_{1, h}\right) .
\end{aligned}
$$

Since $E_{h}$ converges uniformly to $E,\left\|E_{h}\right\|_{\mathcal{H}}$ is uniformly bounded. The conclusion follows upon assigning $u_{h}=E_{h} u$.

When $A$ is unbounded on $\mathcal{H}$ (so that $\mathcal{V} \neq \mathcal{H}$ ), $\left\|P_{h}\right\|_{\mathcal{H}}$ will not typically be uniformly bounded with respect to $h$. Estimating the rate at which $\stackrel{\circ}{\mathcal{H}}_{\mathcal{H}}(h) \rightarrow 0$ 
as $h \rightarrow 0$ becomes technically more demanding and additional hypotheses are honorably acquired. For the remainder of the section we assume that

$$
\mathcal{S}_{1, h}=\mathcal{S}_{2, h} \stackrel{\text { def }}{=} \mathcal{S}_{h}=\operatorname{span}\left\{\phi_{1}, \phi_{2}, \ldots, \phi_{N(h)}\right\}
$$

and that

The trial vectors $\phi_{i}$ are eigenvectors of an auxiliary operator $A_{0}$.

Theorem 6.2. Suppose that $A$ is decomposable as $A=A_{0}+B$ so that $A_{0}$ is positive definite and self-adjoint on $\operatorname{Dom}\left(A_{0}\right)=\operatorname{Dom}(A)$ and $B^{*}$ is bounded relative to $A_{0}$ with relative $A_{0}$-bound less than 1 . Furthermore, assume that $\lambda$ is a nondefective eigenvalue of $A$.

(1) If $B^{*}$ is relatively compact with respect to $A_{0}$, then $\stackrel{\circ}{\mathcal{H}}_{\mathcal{H}}(h) \rightarrow 0$ as $h \rightarrow 0$.

(2) If

$$
\begin{gathered}
\sup _{v \in \mathcal{S}_{h}} \inf _{w \in \mathcal{S}_{h}} \frac{\left\|B^{*} v-w\right\|_{\mathcal{H}}}{\left\|A_{0} v\right\|_{\mathcal{H}}} \stackrel{\text { def }}{=} \stackrel{\circ}{\gamma}(h) \rightarrow 0, \\
\text { then } \stackrel{\circ}{\varepsilon}_{\mathcal{H}}(h) \rightarrow 0 \text { as } h \rightarrow 0 \text { and } \stackrel{\circ}{\varepsilon_{\mathcal{H}}}(h) \leq \mathcal{O}(\stackrel{\circ}{\gamma}(h)) \text { as } h \rightarrow 0 .
\end{gathered}
$$

Proof. Since $B^{*}$ has relative bound with respect to $A_{0}$ smaller than 1 , there is a $\tau \in \mathbb{C}$ so that $\left\|B^{*}\left(A_{0}-\tau\right)^{-1}\right\|_{\mathcal{H}}=1-\kappa<1$ for some $\kappa>0$. It will be useful to translate the spectrum of $A$ by $\tau$ and write $A-\tau=\left(A_{0}-\tau\right)+B$. Referring to the discussion around (3.3), we absorb this shift in spectrum into both $A$ and $A_{0}$, and assume without loss of generality that $\left\|B^{*} A_{0}^{-1}\right\|_{\mathcal{H}}=1-\kappa<1$. Before continuing, we verify that the assumptions of (6.2) and (6.2) are preserved for any appropriate choice of $\tau$. First considering (6.2), if $\tau$ is in the resolvent set for $A_{0}$, then $\tau^{-1}$ is in the resolvent set for $A_{0}^{-1}$ and

$$
B^{*}\left(A_{0}-\tau\right)^{-1}=\frac{1}{\tau} B^{*} A_{0}^{-1}\left(\tau^{-1}-A_{0}^{-1}\right)^{-1} .
$$

$B^{*} A_{0}^{-1}$ is compact, $\left(\tau^{-1}-A_{0}^{-1}\right)^{-1}$ is bounded, so $B^{*}\left(A_{0}-\tau\right)^{-1}$ is also compact and the assumption of (6.2) will be independent of any feasible shift $\tau$. Likewise for (6.2), there is an $m>0$ so that for any $v \in \operatorname{Dom}\left(A_{0}\right),\left\|\left(A_{0}-\tau\right) v\right\|_{\mathcal{H}} \geq m\|v\|_{\mathcal{H}}$. Thus for any $v \in \operatorname{Dom}\left(A_{0}\right)$,

$$
\left\|A_{0} v\right\|_{\mathcal{H}} \leq\left\|\left(A_{0}-\tau\right) v\right\|_{\mathcal{H}}+|\tau| \cdot\|v\|_{\mathcal{H}} \leq\left(1+\frac{|\tau|}{m}\right)\left\|\left(A_{0}-\tau\right) v\right\|_{\mathcal{H}} .
$$

If the assumption of (6.2) holds, then

$$
\sup _{v \in \mathcal{S}_{h}} \inf _{w \in \mathcal{S}_{h}} \frac{\left\|B^{*} v-w\right\|_{\mathcal{H}}}{\left\|\left(A_{0}-\tau\right) v\right\|_{\mathcal{H}}} \leq\left(1+\frac{|\tau|}{m}\right) \stackrel{\circ}{\gamma}(h) \rightarrow 0
$$

so the assumption of (6.2) is independent of the selected shift $\tau$.

Now we prove (6.2) first. Write $A=A_{0}\left(I+A_{0}^{-1} B\right)$ and observe then that

$$
\begin{aligned}
T_{h} Q_{h} A\left(I-Q_{h}\right) T u & =P_{h} T Q_{h} A\left(I-Q_{h}\right) T u \\
& =P_{h} T A_{0} Q_{h} A_{0}^{-1} A\left(I-Q_{h}\right) T u \\
& =P_{h} T A_{0} Q_{h} A_{0}^{-1} B\left(I-Q_{h}\right) T u .
\end{aligned}
$$


Note that

$$
\begin{aligned}
\left\|P_{h} T A_{0} v\right\|_{\mathcal{H}}^{2} & =\left\|\sum_{i, j=1}^{N(h)} a\left(\phi_{j}, T A_{0} v\right) \gamma_{i j} \phi_{i}\right\|_{\mathcal{H}}^{2} \\
& =\sum_{i=1}^{N(h)}\left|\sum_{j=1}^{N(h)}\left\langle\phi_{j}, v\right\rangle_{\mathcal{H}^{\prime}} \lambda_{j}^{0} \gamma_{i j}\right|^{2} \\
& =\|\hat{\mathbf{G}} \mathbf{x}\|_{\mathbb{C}^{N(h)}}^{2},
\end{aligned}
$$

where

$$
\mathbf{x}=\left\{\left\langle\phi_{1}, v\right\rangle_{\mathcal{H}},\left\langle\phi_{2}, v\right\rangle_{\mathcal{H}}, \ldots,\left\langle\phi_{N(h)}, v\right\rangle_{\mathcal{H}}\right\}^{t}
$$

and $\hat{\mathbf{G}}$ is the matrix inverse to

$$
\begin{aligned}
{\left[\left(\lambda_{\ell}^{0}\right)^{-1} a\left(\phi_{\ell}, \phi_{k}\right)\right] } & =\left[\left\langle A_{0}^{-1} \phi_{\ell}, A \phi_{k}\right\rangle_{\mathcal{H}}\right] \\
& =\left[\left\langle\phi_{\ell},\left(I+A_{0}^{-1} B\right) \phi_{k}\right\rangle_{\mathcal{H}}\right] \\
& =\mathbf{I}+\left[\left\langle B^{*} A_{0}^{-1} \phi_{\ell}, \phi_{k}\right\rangle_{\mathcal{H}}\right] .
\end{aligned}
$$

Observe that for any $\mathbf{y} \in \mathbb{C}^{N(h)}$ with $\|\mathbf{y}\|_{\mathbb{C}^{N(h)}}=1$,

$$
\left\|\left[\left\langle B^{*} A_{0}^{-1} \phi_{\ell}, \phi_{k}\right\rangle_{\mathcal{H}}\right] \mathbf{y}\right\|_{\mathbb{C}^{N(h)}} \leq\left\|B^{*} A_{0}^{-1}\right\|_{\mathcal{H}} \leq 1-\kappa,
$$

hence we obtain a bound for $P_{h} T A_{0}$ that is uniform in $h$ :

$$
\left\|P_{h} T A_{0}\right\|_{\mathcal{H}} \leq\|\hat{\mathbf{G}}\|_{\mathbb{C}^{N(h)}} \leq \frac{1}{1-(1-\kappa)}=\frac{1}{\kappa} .
$$

Since $\lambda$ is nondefective, $\lambda T u=u$ for any $u \in \mathcal{U}$ and we find

$$
\begin{aligned}
\stackrel{\circ}{\varepsilon}_{\mathcal{H}}(h) & =\sup _{u \in \mathcal{U}} \frac{\left\|T_{h} Q_{h} A\left(I-Q_{h}\right) T u\right\|_{\mathcal{H}}}{\left\|\left(I-Q_{h}\right) u\right\|_{\mathcal{H}}} \\
& \leq \sup _{u \in \mathcal{U}} \frac{\left\|Q_{h} A_{0}^{-1} B\left(I-Q_{h}\right) T u\right\|_{\mathcal{H}}}{\kappa\left\|\left(I-Q_{h}\right) u\right\|_{\mathcal{H}}} \\
& \leq \sup _{u \in \mathcal{U}} \frac{\left\|Q_{h} A_{0}^{-1} B\left(I-Q_{h}\right) \cdot\left(I-Q_{h}\right) T u\right\|_{\mathcal{H}}}{\kappa|\lambda| \cdot\left\|\left(I-Q_{h}\right) T u\right\|_{\mathcal{H}}} \\
& \leq \frac{1}{\kappa|\lambda|}\left\|Q_{h} A_{0}^{-1} B\left(I-Q_{h}\right)\right\|_{\mathcal{H}} \cdot
\end{aligned}
$$

Now if the condition of (6.2) above holds, then

$$
\left\|Q_{h} A_{0}^{-1} B\left(I-Q_{h}\right)\right\|_{\mathcal{H}} \leq\left\|A_{0}^{-1} B\left(I-Q_{h}\right)\right\|_{\mathcal{H}}=\left\|\left(I-Q_{h}\right) B^{*} A_{0}^{-1}\right\|_{\mathcal{H}} .
$$

Since $B^{*} A_{0}^{-1}$ is compact and $Q_{h} \rightarrow I$ strongly, $\left\|\left(I-Q_{h}\right) B^{*} A_{0}^{-1}\right\|_{\mathcal{H}} \rightarrow 0$ as $h \rightarrow 0$ from which follows the conclusion of (6.2).

Now observe that $A_{0}^{-1} \mathcal{S}_{h}=\mathcal{S}_{h}$ so that, if the assumption (6.2) holds, then

$$
\begin{aligned}
\left\|Q_{h} A_{0}^{-1} B\left(I-Q_{h}\right)\right\|_{\mathcal{H}} & =\left\|\left(I-Q_{h}\right) B^{*} A_{0}^{-1} Q_{h}\right\|_{\mathcal{H}} \\
& =\sup _{u \in \mathcal{S}_{h}} \inf _{w \in \mathcal{S}_{h}} \frac{\left\|B^{*} A_{0}^{-1} u-w\right\|_{\mathcal{H}}}{\|u\|_{\mathcal{H}}} \\
& =\sup _{v \in \mathcal{S}_{h}} \inf _{w \in \mathcal{S}_{h}} \frac{\left\|B^{*} v-w\right\|_{\mathcal{H}}}{\left\|A_{0} v\right\|_{\mathcal{H}}},
\end{aligned}
$$

and as a consequence, $\stackrel{\circ}{\varepsilon}_{\mathcal{H}}(h) \leq \mathcal{O}\left(\stackrel{\circ}{\gamma}^{\prime}(h)\right)$ as $h \rightarrow 0$. 
6.2. Elliptic boundary value problems: Spectral methods. Let $\Omega$ be the unit square in $\mathbb{R}^{2}:[0,1] \times[0,1]$ and let there be given a function $\mathbf{b}=\left[b_{1}(\mathbf{x}), b_{2}(\mathbf{x})\right] \in$ $C^{1}(\Omega) \times C^{1}(\Omega)$ such that $\mathbf{b}=0$ on $\partial \Omega$. Consider the differential operator given by

$$
A(\mathbf{x}, D) u=-\Delta u+\mathbf{b}(\mathbf{x}) \cdot \nabla u+c(\mathbf{x}) u \quad \text { in } \Omega
$$

with $u=0$ on $\partial \Omega$. The regularity results of Kadlec [7, for example, show that $\operatorname{Dom}(A)=H^{2} \cap H_{0}^{1}$.

The application of a Fourier Galerkin method involves trial functions of the form $\phi_{\mathbf{k}}(\mathbf{x})=\sin \left(k_{1} \pi x_{1}\right) \cdot \sin \left(k_{2} \pi x_{2}\right)$. If $|\mathbf{k}|=k_{1}+k_{2}$ denotes the length of the multi-index $\mathbf{k}$, parameterize the family of subspaces as $\mathcal{S}_{h}=\operatorname{span}_{|\mathbf{k}| h<1}\left\{\phi_{\mathbf{k}}\right\}$, and $\operatorname{assign} \mathcal{S}_{h}=\mathcal{S}_{1, h}=\mathcal{S}_{2, h}$. Define $A_{0}=-\Delta$ with $\operatorname{Dom}\left(A_{0}\right)=H^{2}(\Omega) \cap H_{0}^{1}(\Omega)$ and $\operatorname{Dom}\left(A_{0}^{1 / 2}\right)=\mathcal{V}=H_{0}^{1}(\Omega)$. Observe that $A_{0}$ is positive definite and self-adjoint in $\mathcal{H}=L^{2}(\Omega)$ and

$$
A_{0} \phi_{\mathbf{k}}=\lambda_{\mathbf{k}}^{0} \phi_{\mathbf{k}}
$$

with $\lambda_{\mathbf{k}}^{0}=\left(k_{1}^{2}+k_{2}^{2}\right) \pi^{2}$. If $B$ denotes the closure of $\mathbf{b}(\mathbf{x}) \cdot \nabla+c(\mathbf{x})$ on $C_{0}^{\infty}(\Omega)$ then the $\mathcal{H}$-adjoint of $B$ may be calculated as $B^{*} u=-\nabla \cdot(\mathbf{b}(\mathbf{x}) u)=-\mathbf{b}(\mathbf{x}) \cdot \nabla u+(c(\mathbf{x})-$ $\nabla \cdot \mathbf{b}(\mathbf{x})) u$ and $\operatorname{Dom}\left(B^{*}\right) \supset \operatorname{Dom}\left(A_{0}\right)$. Since $B^{*}$ is compact relative to $A_{0}, B^{*}$ has relative $A_{0}$-bound of 0 and Theorem $6.2(1)$ asserts that $\stackrel{\circ}{\mathcal{H}}_{\mathcal{H}}(h) \rightarrow 0$. Furthermore, Theorem 6.2 (2) provides a mechanism for estimating the rate at which $\stackrel{\circ}{\varepsilon}_{\mathcal{H}}(h) \rightarrow 0$.

First, notice that if $\{\lambda, \hat{u}\}$ is an eigenpair for $A$, then

$$
-\Delta \hat{u}=(\lambda-c) \hat{u}-\mathbf{b} \cdot \nabla \hat{u} \in H_{0}^{1}(\Omega)=\operatorname{Dom}\left(A_{0}^{1 / 2}\right)
$$

Thus, $\hat{u} \in \operatorname{Dom}\left(A_{0}^{3 / 2}\right)$ and

$$
\begin{aligned}
\left\|\left(I-Q_{h}\right) \hat{u}\right\|^{2} & =\sum_{|\mathbf{k}| h \geq 1}\left|\left\langle\phi_{\mathbf{k}}, \hat{u}\right\rangle\right|^{2} \\
& =\sum_{|\mathbf{k}| h \geq 1}\left|\left\langle A_{0}^{-3 / 2} \phi_{\mathbf{k}}, A_{0}^{3 / 2} \hat{u}\right\rangle\right|^{2} \\
& =\sum_{|\mathbf{k}| h \geq 1}\left(\lambda_{\mathbf{k}}^{0}\right)^{-3}\left|\left\langle\phi_{\mathbf{k}}, A_{0}^{3 / 2} \hat{u}\right\rangle\right|^{2} \\
& \leq h^{6}\left(\frac{2}{\pi^{2}}\right)^{3} \sum_{|\mathbf{k}| h \geq 1}\left|\left\langle\phi_{\mathbf{k}}, A_{0}^{3 / 2} \hat{u}\right\rangle\right|^{2} \\
& \leq h^{6}\left(\frac{2}{\pi^{2}}\right)^{3}\left\|A_{0}^{3 / 2} \hat{u}\right\|_{\mathcal{H}}^{2}
\end{aligned}
$$

The first inequality is a consequence of $k_{1}^{2}+k_{2}^{2} \geq \frac{1}{2}|\mathbf{k}|^{2} \geq 1 /\left(2 h^{2}\right)$; the second is Bessel's inequality with respect to the orthonormal system $\left\{\phi_{\mathbf{k}}\right\}$.

A similar argument can be organized to estimate $\stackrel{\circ}{\gamma}(h)$. For any $v \in L^{2}(\Omega)$, $A_{0}^{-1} v \in H^{2}(\Omega) \cap H_{0}^{1}(\Omega)$ and $B^{*} A_{0}^{-1} v \in H_{0}^{1}(\Omega) \subset \operatorname{Dom}\left(A_{0}^{1 / 2}\right) . \quad A_{0}^{1 / 2} B^{*} A_{0}^{-1}$ is a closed, everywhere defined operator on $L^{2}(\Omega)$; hence $A_{0}^{1 / 2} B^{*} A_{0}^{-1}$ is a bounded operator on $L^{2}(\Omega)$. 
Now for any $v \in \mathcal{H}$ with $\|v\|_{\mathcal{H}}=1$,

$$
\begin{aligned}
\inf _{w \in \mathcal{S}_{h}}\left\|B^{*} A_{0}^{-1} v-w\right\|_{\mathcal{H}}^{2} & =\sum_{|\mathbf{k}| h \geq 1}\left|\left\langle\phi_{\mathbf{k}}, B^{*} A_{0}^{-1} v\right\rangle\right|^{2} \\
& =\sum_{|\mathbf{k}| h \geq 1}\left|\left\langle A_{0}^{-1 / 2} \phi_{\mathbf{k}}, A_{0}^{1 / 2} B^{*} A_{0}^{-1} v\right\rangle\right|^{2} \\
& =\sum_{|\mathbf{k}| h \geq 1}\left(\lambda_{\mathbf{k}}^{0}\right)^{-1}\left|\left\langle\phi_{\mathbf{k}}, A_{0}^{1 / 2} B^{*} A_{0}^{-1} v\right\rangle\right|^{2} \\
& \leq h^{2}\left(\frac{2}{\pi^{2}}\right) \sum_{|\mathbf{k}| h \geq 1}\left|\left\langle\phi_{\mathbf{k}}, A_{0}^{1 / 2} B^{*} A_{0}^{-1} v\right\rangle\right|^{2} \\
& \leq h^{2}\left(\frac{2}{\pi^{2}}\right)\left\|A_{0}^{1 / 2} B^{*} A_{0}^{-1} v\right\|_{\mathcal{H}}^{2} \leq M h^{2}
\end{aligned}
$$

for some $M<\infty$. From this we obtain

$$
\stackrel{\circ}{\gamma}(h) \leq \sup _{v \in \mathcal{H}} \inf _{w \in \mathcal{S}_{h}} \frac{\left\|B^{*} A_{0}^{-1} v-w\right\|_{\mathcal{H}}}{\|v\|_{\mathcal{H}}} \leq \mathcal{O}(h) .
$$

Theorem 6.1]asserts that there exists an $h_{0}>0$ sufficiently small so that for each $h<h_{0}$ and all $u \in \mathcal{U}$, there is a $u_{h} \in \mathcal{U}_{h}$ so that

$$
\left\|u_{h}-Q_{h} u\right\|_{\mathcal{H}} \leq c h^{4}
$$

whereas $\left\|u_{h}-u\right\|_{\mathcal{H}}$ and $\left\|u-Q_{h} u\right\|_{\mathcal{H}}$ will each be only of order $h^{3}$ in general.

\section{Appendix: Lower Bounds to sep}

Let $\mathcal{W}_{1}$ and $\mathcal{W}_{2}$ be complex Hilbert spaces and denote with $\mathcal{L}\left(\mathcal{W}_{2}, \mathcal{W}_{1}\right)$ the associated Banach space of bounded linear transformations from $\mathcal{W}_{2}$ to $\mathcal{W}_{1}$. Suppose there are given two linear operators, $L_{1}: \mathcal{W}_{1} \rightarrow \mathcal{W}_{1}, L_{2}: \mathcal{W}_{2} \rightarrow \mathcal{W}_{2}$ such that $L_{1}$ is closed, densely defined (but not necessarily bounded) on $\mathcal{W}_{1}$ and $L_{2}$ is bounded (and everywhere defined) in $\mathcal{W}_{2}$. Define an operator $\mathcal{T}: \mathcal{L}\left(\mathcal{W}_{2}, \operatorname{Dom}\left(L_{1}\right)\right) \rightarrow \mathcal{L}\left(\mathcal{W}_{2}, \mathcal{W}_{1}\right)$ as $\mathcal{T}(S)=L_{1} S-S L_{2}$ and let

$$
\operatorname{sep}\left(L_{1}, L_{2}\right) \stackrel{\text { def }}{=} \inf _{S \in \mathcal{L}\left(\mathcal{W}_{2}, D o m\left(L_{1}\right)\right)} \frac{\|\mathcal{T}(S)\|_{\mathcal{W}_{2} \rightarrow \mathcal{W}_{1}}}{\|S\|_{\mathcal{W}_{2} \rightarrow \mathcal{W}_{1}}}
$$

so that, in particular, if $S$ solves $\mathcal{T}(S)=M$ and $0<\eta<\operatorname{sep}\left(L_{1}, L_{2}\right)$, then

$$
\|S\|_{\mathcal{W}_{2} \rightarrow \mathcal{W}_{1}} \leq \frac{1}{\operatorname{sep}\left(L_{1}, L_{2}\right)}\|M\|_{\mathcal{W}_{2} \rightarrow \mathcal{W}_{1}} \leq \frac{1}{\eta}\|M\|_{\mathcal{W}_{2} \rightarrow \mathcal{W}_{1}}
$$

The following results are mild generalizations of [12] Theorem 3.1, p. 264 and [6] Theorem 5, p. 427. For all $\epsilon>0$, we define the pseudospectral sets

$$
\Lambda_{\epsilon}(L)=\left\{z \in \mathbb{C} \mid\left\|(z-L)^{-1}\right\| \geq \frac{1}{\epsilon}\right\}
$$

As $\epsilon \rightarrow 0, \Lambda_{\epsilon}(L)$ shrinks to $\sigma(L)$.

Lemma A.1. Suppose that $\sigma\left(L_{1}\right)$ and $\sigma\left(L_{2}\right)$ are disjoint. Then for every $M \in$ $\mathcal{L}\left(\mathcal{W}_{2}, \mathcal{W}_{1}\right)$, the operator equation

$$
L_{1} S-S L_{2}=M
$$

has a unique solution $S$ that is a bounded linear transformation from $\mathcal{W}_{2}$ to $\operatorname{Dom}\left(L_{1}\right) \subset \mathcal{W}_{1}$. That is, $\mathcal{T}(S)$ is bijective. 
(a) $S$ has the representation

$$
S=\frac{1}{2 \pi \imath} \int_{\Gamma_{2}}\left(L_{1}-z\right)^{-1} M\left(z-L_{2}\right)^{-1} d z,
$$

where $\Gamma_{2}$ consists of a finite number of closed rectifiable Jordan curves enclosing all points of $\sigma\left(L_{2}\right)$ and no points of $\sigma\left(L_{1}\right)$.

(b) Let $\epsilon_{1}, \epsilon_{2}>0$ be chosen so that $\Lambda_{\epsilon_{1}}\left(L_{1}\right) \cap \Gamma_{2}=\emptyset$ and $\Lambda_{\epsilon_{2}}\left(L_{2}\right) \cap \Gamma_{2}=\emptyset$, respectively. That is, $\Lambda_{\epsilon_{1}}\left(L_{1}\right)$ lies entirely outside $\Gamma_{2}$ and $\Lambda_{\epsilon_{2}}\left(L_{2}\right)$ lies entirely inside $\Gamma_{2}$. Then

$$
\operatorname{sep}\left(L_{1}, L_{2}\right) \geq \frac{2 \pi \epsilon_{1} \epsilon_{2}}{\operatorname{length}\left(\Gamma_{2}\right)},
$$

where length $\left(\Gamma_{2}\right)$ is the arc length of $\Gamma_{2}$.

(c) If the numerical ranges of $L_{1}$ and $L_{2}$, denoted respectively $\mathfrak{w}\left(L_{1}\right)$ and $\mathfrak{w}\left(L_{2}\right)$, are disjoint sets in $\mathbb{C}$ and $\Delta=\operatorname{dist}\left(\mathfrak{w}\left(L_{1}\right), \mathfrak{w}\left(L_{2}\right)\right)>0$, then

$$
\operatorname{sep}\left(L_{1}, L_{2}\right) \geq \Delta \text {. }
$$

Proof. A trivial extension of Corollary 3.3 of [12] guarantees that there are a finite number of closed rectifiable Jordan curves enclosing all points of $\sigma\left(L_{2}\right)$ and no points of $\sigma\left(L_{1}\right)$. We orient each of the curves positively (i.e., counterclockwise) and refer to them collectively as $\Gamma_{2}$. Observe that $\Gamma_{2} \subset \rho\left(L_{1}\right) \cap \rho\left(L_{2}\right)$, so the right-hand side of A.3 is well defined and maps $\mathcal{W}_{2}$ into $\operatorname{Dom}\left(L_{1}\right) \subset \mathcal{W}_{1}$. Since $\frac{1}{2 \pi \imath} \int_{\Gamma_{2}}\left(z-L_{2}\right)^{-1} d z=I, \frac{1}{2 \pi \imath} \int_{\Gamma_{2}}\left(z-L_{1}\right)^{-1} d z=0$, and $L(L-z)^{-1}=I+z(L-z)^{-1}$, direct substitution of A.3 into A.2 yields

$$
\begin{aligned}
L_{1} S-S L_{2} & =\frac{1}{2 \pi \imath} \int_{\Gamma_{2}} M\left(z-L_{2}\right)^{-1}-\left(L_{1}-z\right)^{-1} M d z \\
& =M\left(\frac{1}{2 \pi \imath} \int_{\Gamma_{2}}\left(z-L_{2}\right)^{-1} d z\right)-\left(\frac{1}{2 \pi \imath} \int_{\Gamma_{2}}\left(L_{1}-z\right)^{-1} d z\right) M \\
& =M .
\end{aligned}
$$

Suppose $N$ is the difference between any two solutions of (A.2). Then $L_{1} N=N L_{2}$ and

$$
\begin{aligned}
\left(z-L_{1}\right) N & =N\left(z-L_{2}\right) \\
N\left(z-L_{2}\right)^{-1} & =\left(z-L_{1}\right)^{-1} N \\
N\left(\frac{1}{2 \pi \imath} \int_{\Gamma_{2}}\left(z-L_{2}\right)^{-1} d z\right) & =\left(\frac{1}{2 \pi \imath} \int_{\Gamma_{2}}\left(z-L_{1}\right)^{-1} d z\right) N, \\
N & =0 .
\end{aligned}
$$

Thus A.3 gives the one, unique solution to A.2.

To show (b) note that,

$$
\begin{aligned}
\|S\|_{\mathcal{W}_{2} \rightarrow \mathcal{W}_{1}} & \leq \frac{1}{2 \pi} \int_{\Gamma_{2}}\left\|\left(L_{1}-z\right)^{-1}\right\|_{\mathcal{W}_{1}}\|M\|_{\mathcal{W}_{2} \rightarrow \mathcal{W}_{1}}\left\|\left(z-L_{2}\right)^{-1}\right\|_{\mathcal{W}_{2}}|d z| \\
& \leq \frac{\operatorname{length}\left(\Gamma_{2}\right)}{2 \pi} \max _{z \in \Gamma_{2}}\left\|\left(L_{1}-z\right)^{-1}\right\| \mathcal{W}_{1}\|M\|_{\mathcal{W}_{2} \rightarrow \mathcal{W}_{1}} \max _{z \in \Gamma_{2}}\left\|\left(z-L_{2}\right)^{-1}\right\| \mathcal{W}_{2} \\
& \leq \frac{\operatorname{length}\left(\Gamma_{2}\right)}{2 \pi} \frac{1}{\epsilon_{1}} \cdot \frac{1}{\epsilon_{2}}\|M\|_{\mathcal{W}_{2} \rightarrow \mathcal{W}_{1}} .
\end{aligned}
$$

For (c), note that existence and uniqueness of $S$ follows from (a) and the observation that disjoint numerical ranges of $L_{1}$ and $L_{2}$ imply disjoint spectra for $L_{1}$ 
and $L_{2}$. However, we will use a different representation for $S$ to obtain estimates. Define $\hat{z}_{1}, \hat{z}_{2} \in \mathbb{C}$ so that $\hat{z}_{1} \in$ closure $\left\{\mathfrak{w}\left(L_{1}\right)\right\}, \hat{z}_{2} \in \operatorname{closure}\left\{\mathfrak{w}\left(L_{2}\right)\right\}$, and

$$
\begin{aligned}
\Delta=\left|\hat{z}_{1}-\hat{z}_{2}\right| & =\inf _{\substack{z_{1} \in \mathfrak{w}\left(L_{1}\right) \\
z_{2} \in \mathfrak{w}\left(L_{2}\right)}}\left|z_{1}-z_{2}\right| \\
& =\inf _{\substack{x \in \mathcal{W}_{1} \\
y \in \mathcal{W}_{2}}}\left|\frac{\left\langle x, L_{1} x\right\rangle_{\mathcal{W}_{1}}}{\langle x, x\rangle_{\mathcal{W}_{1}}}-\frac{\left\langle y, L_{2} y\right\rangle_{\mathcal{W}_{2}}}{\langle y, y\rangle_{\mathcal{W}_{2}}}\right| .
\end{aligned}
$$

Define $\theta \in[0,2 \pi)$ as $\theta=\arg \left(\hat{z}_{1}-\hat{z}_{2}\right)$. Let $\hat{L}_{1}=e^{-\imath \theta}\left(L_{1}-\hat{z}_{1}\right)$ and $\hat{L}_{2}=$ $e^{-\imath \theta}\left(L_{2}-\hat{z}_{1}\right)$. Then $\mathfrak{w}\left(\hat{L}_{1}\right)=e^{-\imath \theta}\left(\mathfrak{w}\left(L_{1}\right)-\hat{z}_{1}\right)$ and $\mathfrak{w}\left(\hat{L}_{2}\right)=e^{-\imath \theta}\left(\mathfrak{w}\left(L_{2}\right)-\hat{z}_{1}\right)$.

The goal of this translation and rotation is to insure

$$
\Re e\left\langle x, \hat{L}_{1} x\right\rangle_{\mathcal{W}_{1}} \geq 0
$$

for all $x \in \operatorname{Dom}\left(L_{1}\right)$, and

$$
\Re e\left\langle y, \hat{L}_{2} y\right\rangle_{\mathcal{W}_{2}} \leq-\Delta\|y\|_{\mathcal{W}_{2}}^{2}
$$

for all $y \in \mathcal{W}_{2}$.

Under these circumstances, the Lumer-Phillips theorem (cf. [11], §1.4) guarantees that $-\hat{L}_{1}$ and $\hat{L}_{2}$ each generate strongly continuous, one parameter semigroups, $e^{-t \hat{L}_{1}}$ and $e^{t \hat{L}_{2}}$, respectively. Furthermore, $\left\|e^{-t \hat{L}_{1}}\right\|_{\mathcal{W}_{1}} \leq 1$ and $\left\|e^{t \hat{L}_{2}}\right\|_{\mathcal{W}_{2}} \leq e^{-t \Delta}$ for all $t \geq 0$.

Notice that $S$ satisfies (A.2) if and only if it is also a solution to $\hat{L}_{1} S-S \hat{L}_{2}=$ $e^{-\imath \theta} M$ and this leads to the following representation for $S$ :

$$
S=e^{-\imath \theta} \int_{0}^{\infty} e^{-t \hat{L}_{1}} M e^{t \hat{L}_{2}} d t .
$$

Indeed, note that with this expression for $S$, we have for any $v \in \mathcal{W}_{2}$

$$
\begin{aligned}
\|\left(L_{1} S\right. & \left.-S L_{2}-M\right) v \|_{\mathcal{W}_{1}} \\
= & \left\|\left(\hat{L}_{1} S-S \hat{L}_{2}-e^{-\imath \theta} M\right) v\right\|_{\mathcal{W}_{1}} \\
= & \left\|\int_{0}^{\infty} \hat{L}_{1} e^{-t \hat{L}_{1}} M e^{t \hat{L}_{2}} v-e^{-t \hat{L}_{1}} M e^{t \hat{L}_{2}} \hat{L}_{2} v d t-M v\right\|_{\mathcal{W}_{1}} \\
= & \left\|\int_{0}^{\infty}-\frac{d}{d t}\left(e^{-t \hat{L}_{1}} M e^{t \hat{L}_{2}} v\right) d t-M v\right\|_{\mathcal{W}_{1}} \\
= & \| \lim _{t \rightarrow 0}\left(e^{-t \hat{L}_{1}} M v-M v\right) \\
& \quad+\lim _{t \rightarrow 0} e^{-t \hat{L}_{1}} M\left(e^{t \hat{L}_{2} v-v} v\right)-\lim _{t \rightarrow \infty}\left(e^{-t \hat{L}_{1}} M e^{t \hat{L}_{2}} v\right) \|_{\mathcal{W}_{1}} \\
\leq & \lim _{t \rightarrow 0} \|\left(e^{-t \hat{L}_{1}} M v-M v\left\|_{\mathcal{W}_{1}}+\right\| M\left\|_{\mathcal{W}_{2} \rightarrow \mathcal{W}_{1}} \lim _{t \rightarrow 0}\right\| e^{t \hat{L}_{2}} v-v \|_{\mathcal{W}_{1}}\right. \\
& +\lim _{t \rightarrow \infty}\|M\|_{\mathcal{W}_{2} \rightarrow \mathcal{W}_{1}} e^{-t \Delta}\|v\|_{\mathcal{W}_{2}}=0 .
\end{aligned}
$$

Immediately then, one obtains

$$
\begin{aligned}
\|S\|_{\mathcal{W}_{2} \rightarrow \mathcal{W}_{1}} & \leq \int_{0}^{\infty}\left\|e^{-t \hat{L}_{1}}\right\|_{\mathcal{W}_{1}}\|M\|_{\mathcal{W}_{2} \rightarrow \mathcal{W}_{1}}\left\|e^{t \hat{L}_{2}}\right\|_{\mathcal{W}_{2}} d t \\
& \leq \int_{0}^{\infty} e^{-t \Delta} d t\|M\|_{\mathcal{W}_{2} \rightarrow \mathcal{W}_{1}}=\left(\frac{1}{\Delta}\right)\|M\|_{\mathcal{W}_{2} \rightarrow \mathcal{W}_{1}} .
\end{aligned}
$$




\section{ACKNOWLEDGMENTS}

I have had the pleasure of stimulating correspondence and constructive critique from both Professor John Osborn of the University of Maryland at College Park and Professor Andrew Knyazev of the University of Colorado at Denver.

\section{REFERENCES}

[1] Babuška, I., and J. Osborn (1991), Eigenvalue Problems, in "Finite Element Methods" Handbook of Numerical Analysis, Vol. 2, edited by P. G. Ciarlet and J. L. Lions. Elsevier Science Publisher (North Holland). CMP 91:14

[2] Babuška, I., and J. Osborn (1989), Finite element-Galerkin approximation of the eigenvalues and eigenvectors of selfadjoint problems, Math. Comp. 52, pp. 275-297. MR 89k:65132

[3] Chatelin, F. (1983), Spectral Approximation of Linear Operators, (Academic Press, New York) MR 86d:65071

[4] Descloux, J., N. Nassif, and J. Rappaz (1978), On spectral approximation, Part I: The problem of convergence, RAIRO Anal. Numér. 12, pp. 97-112. MR 58:3404a

[5] Descloux, J., M. Luskin, and J. Rappaz (1981), Approximation of the spectrum of closed operators: the determination of normal modes of a rotating basin, Math. Comp. 36, pp. 137-154. MR 83h:65123

[6] Heinz, E. (1951), Beiträge zur Störungstheorie der Spektralzerlegung, Math. Ann. 123, pp. 415-438. MR 13:471f

[7] Kadlec, J. (1964), The regularity of the solution of the Poisson problem in a domain whose boundary is similar to that of a convex domain, Czech Math J. 14 (89), pp. 386-393. (In Russian) MR 30:329

[8] Kato, T. (1960), Estimation of iterated matrices, with application to the von Neumann condition, Numerische Mathematik 2, pp. 22-29. MR 22:711

[9] Kato, T. (1976), Perturbation Theory for Linear Operators, (Springer, Heidelberg) MR 53:11389

[10] Knyazev, A. (1997), New estimates for Ritz vectors, Math. Comp., 66 (219), pp. 985-995. MR 97j:65090

[11] Pazy, A. (1983), Semigroups of Linear Operators and Applications to Partial Differential Equations, (Springer, Heidelberg) MR 85g:47061

[12] Rosenblum, M. (1956), On the operator equation $B X-X A=Q$, Duke Math. J. pp. 263-269. MR 18:54d

[13] Wloka, J. (1987), Partial Differential Equations, (Cambridge Univ. Press, Cambridge) MR 88d:35004

Department of Mathematics, Virginia Polytechnic Institute and State University, BLACKSBURG, VA 24061 USA

E-mail address: beattie@math.vt.edu 